\title{
PLANTAS VASCULARES DE LOS RIOS BAKER Y PASCUA, REGION DE AISEN, CHILE
}

\author{
VASCULAR PLANTS OF BAKER AND PASCUA RIVERS, REGION OF AISEN, \\ CHILE
}

\author{
Roberto Rodríguez, Alicia Marticorena \& Ernesto Teneb \\ Departamento de Botánica, Universidad de Concepción, Chile \\ rrodrigu@udec.cl
}

\section{RESUMEN}

\begin{abstract}
Se reportan los resultados de las excursiones botánicas realizadas durante los años 2006- 07 a las cuencas de los ríos Baker y Pascua en la Región de Aisén, Patagonia chilena. Se encontraron 342 taxa de plantas vasculares, 302 en la cuenca del río Baker y 124 en río Pascua. Las familias mejor representadas son Poaceae (52), Asteraceae (50) y Cyperaceae (15). Cuatro especies endémicas de Chile fueron encontradas en la cuenca del río Baker.
\end{abstract}

Palabras claves: Flora de Chile, Patagonia chilena, conservación, plantas invasoras.

\section{ABSTRACT}

The results of the excursion made during 2006-07 to the basins of Baker and Pascua rivers on the Chilean Patagonia are reported. There are 342 taxa of vascular plants, 302 on the Baker River, and 124 on the Pascua River. The families with higher numbers were Poaceae (52), Asteraceae (50) and Cyperaceae (15). Four endemics species were found on the basin of Baker River.

Keywords: Flora of Chile, Chilean Patagonia, conservation, alien plants.

\section{INTRODUCCION}

Los ríos Baker y Pascua se ubican en las provincias de Cochrane y Capitán Prat respectivamente, y pertenecen a la XI Región de Aisén del General Carlos Ibáñez del Campo. El río Baker nace en el lago Bertrand (46'59'00''S; 72 50'51''W), el que recorre $197 \mathrm{~km}$ para finalmente desembocar en el

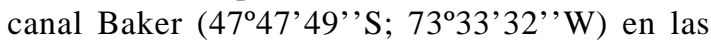
proximidades de Caleta Tortel. En tanto que el río Pascua, descubierto por Hans Steffen en 1898, se origina en el lago O'Higgins (48 $20^{\prime} 21^{\prime}$ 'S $\left.73^{\circ} 00^{\prime} 41^{\prime \prime}, \mathrm{W}\right)$, que tras recorrer $67 \mathrm{~km}$ desemboca en el fiordo Steele (48 $13^{\circ} 32^{\prime}$ 'S; 73 $\left.18^{\circ} 51^{\prime \prime} \mathrm{W}\right)$. La vegetación del área está dominada por bosques siempreverdes, bosques deciduos y turberas, las especies dominantes del paisaje son representantes de la familia Nothofagaceae comoNothofagus nitida (Phil.) Krasser, N. betuloides (Mirb.) Oerst., N. pumilio (Poepp. et Endl.) Krasser y N. antarctica (G.Forst.) Oerst. También son importantes Pilgerodendron uviferum (D.Don) Florin,Tepualia stipularis (Hook. et Arn.) Griseb.,Amomyrtus luma (Molina) D.Legrand et Kausel y Podocarpus nubigena Lindl.; entre los arbustos son abundantes: Lepidothamnus fonkii Phil., Berberis microphylla GForst. y diversas especies de Escallonia. En las zonas xéricas dominan arbustos como Gaultheria mucronata (L.f.) Hook. et Arn., Colletia hystrix Clos y Mulinum spinosum (Cav.) Pers. (Reiche 1907, Gajardo 1994, Luebert y Pliscoff 2006).

Desde los comienzos del siglo XX se han publicado resultados de excursiones botánicas a la 
patagonia occidental y Aisén (Turrill 1919, Skottsberg 1921, Espinosa 1935, Hambleton 1936, Looser 1945, Gunckel 1970, Pisano 1972 y 1988, Teillier y Marticorena 2002), o estudios fitogeográficos que tratan el territorio patagónico y regiones adyacentes (Neger 1901, Skottsberg 1910, Pisano 1981), o sobre alcances generales de la vegetación, su origen y descripción de ella (Dusén 1903, Macloskie 1903-06, Dusén \& Neger 1908, Macloskie \& Dusén 1915, Skottsberg 1916, Skottsberg 1923, Seki 1968).

El único trabajo de flora relacionado directamente con el río Baker es el de Hambleton (l.c.), quien participó en una expedición en diciembre de 1898 acompañando a geógrafos de la Comisión de Límites de Chile y Argentina (Steffen 1904), que entonces recorrieron desde la desembocadura del río Baker hasta el lago Cochrane. Allí se mencionan 148 taxones de plantas vasculares, de las cuales 142 son nativas y 6 son introducidas. No existen, hasta ahora, antecedentes botánicos para el río Pascua, por lo que este catálogo constituye el primer registro que se tiene para esa área en particular.

Actualmente se ha incrementado el interés por el conocimiento de las plantas vasculares de la Región de Aisén, y es así como se encuentran en desarrollo proyectos enfocados hacia la biodiversidad (Torres-Mura \& Rojas 2004), que en el futuro entregarán antecedentes concretos para la toma de decisiones en el uso de los recursos naturales del país. El objetivo del presente artículo es reportar una lista preliminar de la flora vascular, de las cuencas de los ríos Baker y Pascua, incluyendo comentarios de origen biogeografico, hábito y distribución en el país.

\section{MATERIALES Y METODOS}

Durante los años 2006 y 2007 se realizaron cinco campañas a la zona de estudio, enmarcadas en el estudio de Línea Base de Flora y Vegetación del Proyecto Hidroeléctrico Aisén, lo que permitió visitar más de 200 sitios repartidos en ambas cuencas (Fig. 1). Mediante este estudio de línea base se generó un listado preliminar de la flora vascular presente en el área. Las plantas recolectadas fueron herborizadas y están depositadas en el Herbario de la Universidad de Concepción (CONC). El estado de conservación de las especies se basó en: Red List UICN (Walter \& Gillett 1998), Hechenleitner (2005) y en las listas entregadas por el Boletín 47 del Museo de Historia Natural de Chile (Baeza et al. 1998). La nomenclatura sigue a Cronquist (1981), Marticorena \& Quezada (1985), Matthei (1995) y Rodríguez et al. (2005). La información sobre el origen está basada en Parodi (1959) y la condición de maleza en Matthei (l.c.).

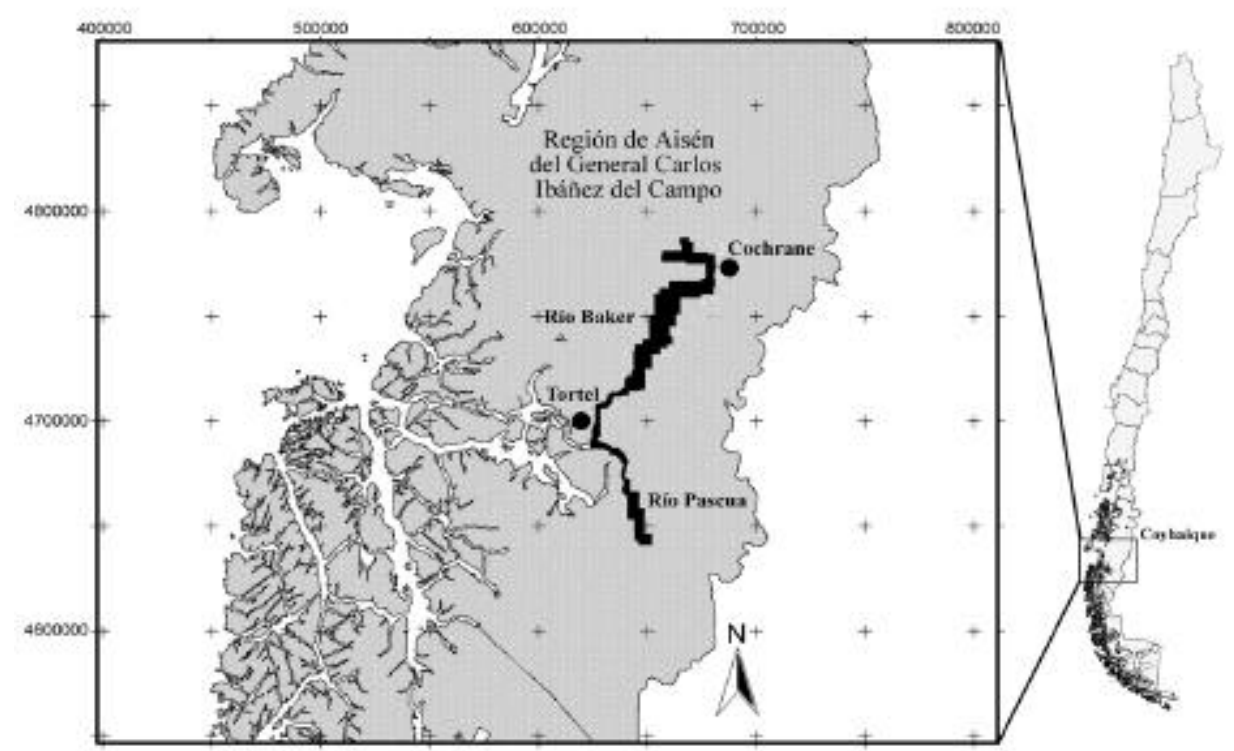

FiguRa 1. Ubicación geográfica de las cuencas de los ríos Baker y Pascua.

FIGURE 1. Geographic localization of the basins Baker and Pascua rivers. 
Los comentarios sobre la abundancia, están basados en las apreciaciones hechas por los autores, entendiendo ello como comentarios generales, con el fin de graficar mejor la situación de abundancia de cada especie. Las categorías usadas fueron Escasa, Frecuente y Abundante, que señalan, en forma progresiva, mayor densidad de individuos de una determinada especie.

En las especies señaladas C. Ramírez*, corresponde a los materiales depositados en el Instituto de Biología de la Universidad Austral de Chile, que se perdieron en el incendio en diciembre de 2007.

\section{RESULTADOS}

ANALISIS GENERALES

En las cuencas de los ríos Baker y Pascua se encontraron 342 taxa de plantas vasculares, dentro de 85 familias, donde las más numerosas son
Poaceae (52), Asteraceae (50), Cyperaceae (15), Apiaceae (10) y Rosaceae (13). La cuenca del río Baker muestra mayor riqueza en los tres niveles taxonómicos analizados (Fig. 2). La cuenca del río Baker presenta 302 especies, mientras que la cuenca del río Pascua tiene 124 especies de plantas vasculares. Estas recolecciones constituyen una primera aproximación al conocimiento de la diversidad vegetal del área.

En la cuenca del río Baker, el grupo mejor representado es Magnoliopsida con 200 especies, seguido por Liliopsida con 80. Un patrón similar se observa en la cuenca del Pascua. La cuenca del Baker presenta mayor riqueza de especies en la mayoría de los grupos taxonómicos excepto en Pteridophyta, donde la cuenca del Pascua presenta 20 especies contra 16 del río Baker (Tabla I).

Por condición biológica o hábito, las plantas vasculares más frecuentes en ambas cuencas son las hierbas perennes con 207 taxa, seguidas por las hierbas anuales con 53 taxa (Tabla II).

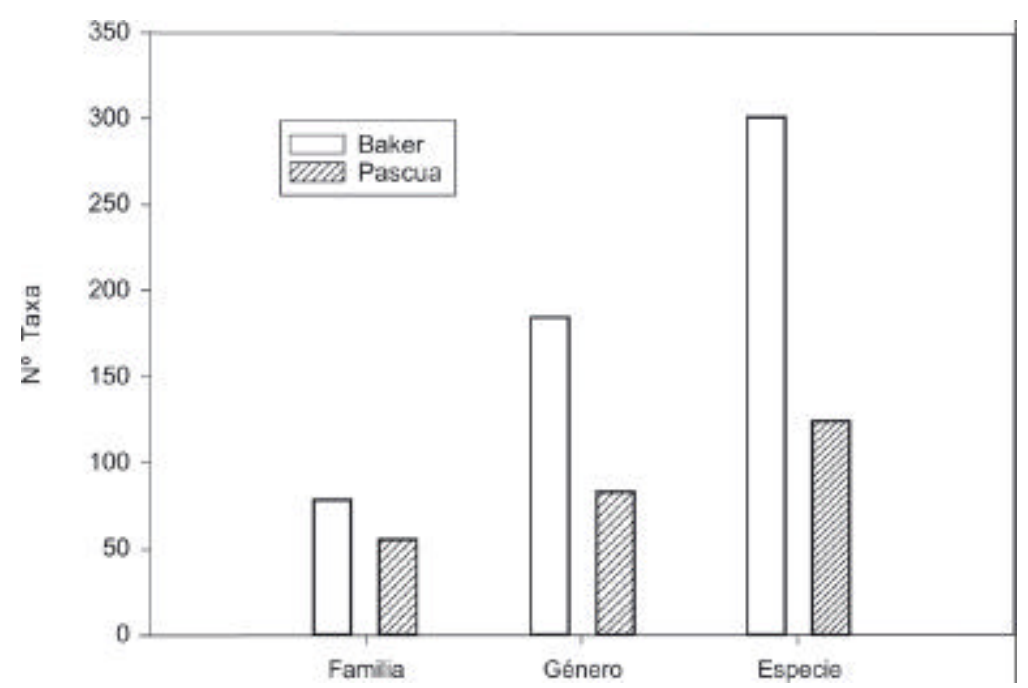

Figura 2. Distribución de riqueza de especies por nivel taxonómico en las cuencas de los ríos Baker y Pascua.

FIGURE 2. Richness distribution by taxonomic level in the basins Baker and Pascua Rivers.

TABLA I. Riqueza de especies por grupo taxonómico en las cuencas de los ríos Baker y Pascua

TABLE I. Richness of each taxonomic group in the basins Baker and Pascua Rivers.

\begin{tabular}{lccccc}
\hline & Pteridophyta & Pinophyta & Magnoliopsida & Liliopsida & Total \\
\hline Río Baker & 16 & 6 & 200 & 80 & 302 \\
Río Pascua & 20 & 3 & 79 & 22 & 124 \\
Especies compartidas & 9 & 3 & 57 & 15 & 84 \\
\hline
\end{tabular}


Si se considera el origen biogeográfico de las especies, en ambas cuencas la mayoría son nativas, solamente en la cuenca del río Baker se encontraron especies endémicas (Tabla II). Por otra parte, las especies introducidas son más frecuentes en la cuenca del río Baker, representadas por 68 taxa, ello debido a la alta presión antrópica a la que ha estado sometida dicha cuenca. Una situación muy distinta muestra la cuenca del río Pascua, con sólo 4 especies introducidas, condición debido al difícil acceso y al aislamiento geográfico (distancia a caminos y centros poblados), lo que redunda en una baja intervención.

En ambas cuencas estudiadas, las plantas más frecuentes son las hierbas perennes nativas, con 144 taxa en el Baker y 73 taxa en el Pascua, seguido por los arbustos, también nativos con 34 y 25 taxa respectivamente (Tabla II).

De las especies endémicas encontradas, ninguna presenta problemas de conservación. El árbol endémico Nothofagus nitida presenta un rango de distribución desde la Región de Los Lagos a la de Magallanes; es abundante en tierras bajas de la parte sur de la cuenca del río Baker. El resto de las especies endémicas presentan una amplia distribución, llegando incluso a la zona de clima mediterráneo en Chile central.

En el área de estudio se encontraron 14 (3,9\%) especies de plantas vasculares en categorías de conservación (Tabla III), de ellas 9 son Pteridophyta, 3 son Pinophyta y 2 son Liliopsida.

La única especie catalogada EN PELIGRO fue Herbertia lahue (Molina) Goldblatt, que es una Iridaceae que en Chile crece desde la Región de Valparaíso a la Región de Los Lagos; ésta fue encontrada en las cercanías de Lago Esmeralda. Con este hallazgo se extiende el rango de distribución de esta especie y del género en $3^{\circ}$ de latitud. Se encontraron, además, ocho especies en categoría VULNERABLE, dos especies en categoría RARA y dos especies en categoría INSUFICIENTEMENTE CONOCIDA. Se ha propuesto a Arachnitis uniflora Phil. como especie VULNERABLE debido a la alteración del hábitat, y a que se encuentra en poblaciones pequeñas y aisladas a lo largo de su distribución.

Este estudio permitió determinar dos nuevos registros de especies para el país, Jarava nana (Speg.) Peñail. y Lupinus polyphyllus Lindl.

TABLA II. Riqueza de especies por origen biogeográfico y condición biológica para las cuencas de los ríos Baker y Pascua. N: nativa, E: endémica, I: introducida.

TABLE II. Richness by origin biogeographic and habitus in the basins Baker and Pascua Rivers. N: native, E: endemic, I: alien.

\begin{tabular}{lccccccc}
\hline \multirow{2}{*}{ Hábito } & Total & \multicolumn{3}{c}{ Río Baker } & \multicolumn{3}{c}{ Río Pascua } \\
& por hábito & N & E & I & N & E & I \\
\hline Árbol & 20 & 14 & 1 & 5 & 11 & - & - \\
Arbusto & 42 & 34 & 1 & 3 & 25 & - & - \\
Subarbusto & 20 & 16 & - & - & 10 & - & - \\
Hierba perenne & 207 & 144 & 1 & 30 & 73 & - & 2 \\
Hierba anual & 53 & 22 & 1 & 30 & 1 & - & 2 \\
\hline Total & 342 & 230 & 4 & 68 & 120 & 0 & 4 \\
\hline Total cuenca & & & 302 & & & 124 & \\
\hline
\end{tabular}


Plantas vasculares de los ríos Baker y Pascua: RodríGuEz, R. ET AL.

Tabla III. Especies en categoría de conservación y su distribución en Chile.

Table III. Species conservation category and its distribution in Chile.

\begin{tabular}{llcc}
\hline Familia & \multicolumn{1}{c}{ Especie } & Estado de conservación $^{\text {Distribución en Chile }}$ \\
\hline Iridaceae & Herbertia lahue & En Peligro $^{4}$ & V - XI \\
Corsiaceae & Arachnitis uniflora & Vulnerable $^{3}$ & VII - XII \\
Cupressaceae & Pilgerodendron uviferum & Vulnerable $^{2,4}$ & X - XII \\
Grammitidaceae & Grammitis magellanica & Vulnerable $^{1}$ & VIII - XII \\
Hymenophyllaceae & Hymenophyllum secundum & Vulnerable $^{1}$ & X - XII \\
Hymenophyllaceae & Hymenophyllum tortuosum & Vulnerable $^{1}$ & X - XII \\
Lycopodiaceae & Lycopodium paniculatum & Vulnerable $^{1}$ & VIII - XI \\
Podocarpaceae & Lepidothamnus fonkii & Vulnerable $^{4}$ & X - XII \\
Podocarpaceae & Podocarpus nubigena & Vulnerable $^{4}$ & IX - XII \\
Schizaeaceae & Schizaea fistulosa & Vulnerable $^{1}$ & X - XII \\
Lycopodiaceae & Lycopodium confertum & Rara $^{1}$ & X - XII \\
Lycopodiaceae & Lycopodium gayanum & Rara $^{1}$ & VIII - XI \\
Dennstaedtiaceae & Hypolepis poepigii & Ins. Conocida $^{1}$ & IV - XII \\
Hymenophyllaceae & Serpyllopsis caespitosa & Ins. Conocida $^{1}$ & X - XII \\
\hline
\end{tabular}

${ }^{1}$ Baeza et al. 1998; ${ }^{2}$ Hechenleitner et al. 2005; ${ }^{3}$ Rodríguez et al., en este trabajo; ${ }^{4}$ Walter \& Gillett 1998 .

\section{CATÁlogo Comentado PTERIDOPHYTA}

\section{Adiantaceae}

Adiantum sulphureum Kaulf.

Hierba perenne, nativa de Chile y Argentina. En Chile se distribuye entre las regiones de Coquimbo y Aisén. Escaso en la cuenca del Baker. Exs.: Cochrane. El Salto, Valle Grande. R. Rodríguez y E. Teneb 4207 (CONC 166397).

Cheilanthes glauca (Cav.) Mett. "doradilla" Hierba perenne, nativa de Chile y Argentina. Se distribuye entre las regiones de Coquimbo y Aisén. Frecuente en la cuenca del Baker. Exs.: Río Colonia. Sector La Península. R. Rodríguez y E. Teneb 4254 (CONC 166463).

\section{Aspleniaceae}

Asplenium dareoides Desv. "filulawen" Hierba perenne, epífita, nativa de Chile y Argentina. Se distribuye entre las regiones de Coquimbo y de Magallanes. Frecuente en la cuenca del Pascua. Exs.: Entre Lago Leal y Quetru. R. Rodríguez y E. Teneb 4173 (CONC 166416).

\section{Blechnaceae}

Blechnum chilense (Kaulf.) Mett.

"costilla de vaca"

Hierba perenne, nativa de Chile y Argentina. Se encuentra desde la Región de Coquimbo hasta la de Magallanes. Frecuente en bosques siempreverdes de ambas cuencas. Exs.: Río Baker. El Saltón. R. Rodríguez y E. Teneb 4282 (CONC 166432).

Blechnum magellanicum (Desv.) Mett. "catalapi" Hierba perenne, nativa de Chile y Argentina. Se encuentra desde la Región del Maule hasta la de Magallanes. Frecuente en bosques siempreverdes de la cuenca del Pascua. Exs.: Río Pascua. E. Teneb 163 (CONC 166433).

Blechnum penna-marina (Poir.) Kuhn "pasto serrucho, punque" Hierba perenne, nativa de Chile y Argentina. En Chile se encuentra en las regiones de Aisén y Magallanes. Abundante en ambas cuencas. Exs.: Río Baker. El Saltón. R. Rodríguez y E. Teneb 4289 (CONC 166434).

\section{Dennstaedtiaceae}

Hypolepis poeppigii (Kunze) R.A.Rodr.

"wilel-lawén"

Hierba perenne, nativa de Chile y Argentina. Se distribuye entre la Región de Coquimbo y la de Magallanes. Escasa en la cuenca del Pascua. Estado de conservación: Insuficientemente Conocida (Baeza et al. 1998). Exs.: Entre Lago Leal y Quetru. R. Rodríguez y E. Teneb 4168 (CONC 166553). 


\section{DRYOPTERIDACEAE}

Polystichum andinum Phil.

Hierba perenne, nativa de Chile y Argentina. Se distribuye desde la Región de O'Higgins hasta la de Magallanes. Escaso en la cuenca del Baker. Exs.: Cochrane. Puerto Bertrand. C. Ramírez y O. Vidal s/n (CONC 166638).

Polystichum multifidum (Mett.) T.Moore

Hierba perenne, nativa de Chile y Argentina. Se encuentra desde la Región de Los Lagos hasta la de Magallanes. Escaso en la cuenca del Pascua. Exs.: Entre Lago Leal y Quetru. R. Rodríguez y E. Teneb 4165 (CONC 166639).

Polystichum plicatum (Poepp. ex Kunze) Hicken Hierbaperenne, nativa de Chiley Argentina. Se distribuye entre las regiones de Coquimbo y la de Magallanes. Escasa en la cuenca del Baker. Exs.: Río Baker. El Saltón. R. Rodríguez y E. Teneb 4290 (CONC 166640).

Rumohra adiantiformis (G.Forst.) Ching "pereq" Hierba perenne, nativa de Chile y Argentina. Se distribuye entre las regiones de Coquimbo y la de Magallanes. Escasa en la cuenca del Baker. Exs.: Río Baker. El Saltón. R. Rodríguez y E. Teneb 4287 (CONC 166660).

\section{GLEICHENIACEAE}

Gleichenia cryptocarpa Hook. "hierba loza" Hierba perenne, nativa en Chile y Argentina. Se encuentra entre las regiones del Bío-Bío y la de Magallanes. Escasa en ambas cuencas. Exs.: Tortel, parte alta, en una turbera. R.Rodríguez y E. Teneb 4078 (CONC 166530).

\section{Gleichenia quadripartita (Poir.) T.Moore}

"hierba loza" Hierba perenne, nativa de Chile y Argentina. En Chile se distribuye entre las regiones del Bío-Bío y Magallanes. Frecuente en ambas cuencas. Exs.: Entre Lago Leal y Quetru. R. Rodríguez y E. Teneb 4163 (CONC 166531).

\section{Grammitis magellanica Desv.}

Hierba perenne, epífita, nativa de Chile y Argentina. Se encuentra entre las regiones del Bío-Bío y la de Magallanes. Frecuente en la cuenca del Pascua. Estado de conservación Vulnerable (Baeza et al . 1998). Exs.: Río Pascua. Pampa Quiroz. E. Teneb y A. Jiménez 4241 (CONC 166533).

\begin{abstract}
HyMenophyllaceAe
Hymenophyllum dentatum Cav. "susu-lahuén" Hierba perenne, epífita, nativa de Chile y Argentina. Su distribución en Chile va desde la Región del BíoBío a la de Magallanes. Frecuente en los bosques de la cuenca del Pascua. Exs.: Puerto Yungay. A. Jiménez 101 (CONC 166546).
\end{abstract}

\section{Hymenophyllum krauseanum Phil.}

Hierba perenne, epífita, nativa de Chile y Argentina. Su distribución en Chile es desde la Región del BíoBío hasta la de Aisén. Es una especie frecuente en los bosques de la cuenca del Pascua. Exs.: Entre Lago Leal y Quetru. R. Rodríguez y E. Teneb 4172 (CONC 166547).

\section{Hymenophyllum pectinatum Cav.}

Hierba perenne, epífita, nativa de Chile y Argentina. Se distribuye entre las regiones de la Araucanía y la de Magallanes. Abundante en los bosques de fagáceas de la cuenca del Pascua. Exs.: Entre Lago Leal y Quetru. R. Rodríguez y E. Teneb 4171 (CONC 166548).

Hymenophyllum secundum Hook. et Grev.

Hierba perenne, epífita, nativa de Chile y Argentina. Se distribuye entre las regiones de Los Lagos y la de Magallanes. Frecuente en los bosques de nothofagáceas de ambas cuencas. Estado de conservación: Vulnerable (Baeza et al. 1998). Exs.: Cochrane. Lago Vargas. E. Teneb 71 (CONC 166549).

Hymenophyllum tortuosum Hook. et Grev.

Hierba perenne, epífita, nativa de Chile y Argentina. Se distribuye entre la Región de Los Lagos y la de Magallanes. Frecuente en los bosques de la cuenca del Pascua. Estado de conservación: Vulnerable (Baeza et al. 1998). Exs.: Río Pascua. Angostura San Vicente. R. Rodríguez y E. Teneb 4183 (CONC 166550).

Serpyllopsis caespitosa (Gaudich) C.Chr.

Hierba perenne, nativa de Chile y Argentina. Se distribuye desde la Región de Los Lagos hasta la de Magallanes. Escasa en los bosques de la cuenca del Pascua. Estado de conservación: InsuFICIENTEMENTE Conocida (Baeza etal. 1998). Exs.: Sector Vagabundo. A. Jiménez 129 (CONC 166682).

\section{LyCOPODIACEAE}

Lycopodium alboffii Rolleri

Hierba perenne, nativa de Chile y Argentina. Su 
distribución en Chile es desde la Región de Los Lagos a la de Magallanes. Especie frecuente en laderas rocosas y expuestas de la cuenca del Pascua. Exs.: Río Pascua. Pampa Quiroz. E. Teneb y A. Jiménez 4250 (CONC 166574).

Lycopodium confertum Willd.

Hierba perenne, nativa de Chile y Argentina. Se distribuye entre las regiones de Los Lagos y Magallanes. Es una especie frecuente en las cuencas de los ríos Baker y Pascua. Estado de conservación: RARA (Baeza et al. 1998). Exs.: Tortel. Cerros frente al delta. R. Rodríguez y E. Teneb 4205 (CONC 166575).

\section{Lycopodium gayanum J.Remy}

Hierba perenne, nativa de Chile y Argentina. Se distribuye entre las regiones del Bío-Bío y la de Magallanes. Escasa en ambas cuencas. Estado de conservación: RARA (Baeza et al. 1998). Exs.: Tortel, parte alta, en una turbera. R.Rodríguez y E. Teneb 4075 (CONC 166576).

\section{Lycopodium magellanicum (P.Beauv.) Sw.}

Hierba perenne, nativa de Chile y Argentina. Se encuentra desde la Región del Bío-Bío a a la de Magallanes. Frecuente en ambas cuencas. Exs.: Camino de Cochrane a Tortel. El Barrancoso. R. Rodríguez y E. Teneb 4127 (CONC 166577).

\section{Lycopodium paniculatum Desv.}

"Palmita, pimpinela" Hierba perenne, nativa de Chile y Argentina. Se encuentra desde la Región del Bío-Bío a la de Magallanes. Frecuente en ambas cuencas. Estado de conservación: Vulnerable (Baeza et al. 1998). Exs.: Camino de Cochrane a Tortel. El Barrancoso. R. Rodríguez y E. Teneb 4126 (CONC 166578).

\section{Schizaea fistulosa Labill.}

\section{Schizaeaceae}

Hierba perenne, nativa de Chile, cosmopolita. Se distribuye entre la Región de Los Lagos y la de Magallanes. Escasa en la cuenca del Baker. Estado de conservación: Vulnerable (Baeza et al. 1998). Exs.: Caleta Tortel. R. Vargas 431 (CONC 166666).

\section{W OODSIACEAE}

Cystopteris fragilis (L.) Bernh. var. apiiformis (Gand.) C.Chr.

Hierba perenne, nativa de Chile, cosmopolita. Se distribuye entre las regiones de Tarapacá y la de
Magallanes. Escasa en la cuenca del Baker. Exs.: Cochrane. El Salto, Valle Grande. R. Rodríguez y E. Teneb 4208 (CONC 166479).

\section{PINOPHYTA}

\section{Cupressaceae}

Pilgerodendron uviferum (D.Don) Florin "ciprés de las Guaitecas" Arbol nativo de Chile y Argentina. Se distribuye entre la Región de Los Lagos y la de Magallanes. Especie frecuente en el sector sur de la cuenca del río Baker y abundante en la cuenca del Pascua. Estado de conservación: VulNERABLE (Walters \& Guillett 1998). Exs.: Entre Lago Leal y Quetru. R. Rodríguez y E. Teneb 4160 (CONC 166623).

\section{PinACEAe}

Larix decidua Miller "alerce europeo" Arbol introducido en Chile, originario del centro de Europa. Es cultivado con fines ornamentales y forestales. Esta especie fue hallada en el sector angostura Tamango en la cuenca del Baker. Exs.: Cochrane. Ladera del cerro Tamango. R. Rodríguez y E. Teneb 4114 (CONC 166560).

Pinus contorta Douglas ex Loudon

"pino costero" Arbol introducido en Chile, originario de Norteamérica. En Chile es cultivado con fines forestales. Esta especie ha sido introducida en la cuenca del Baker. Exs.: Sector Lago Chacabuco. E. Teneb 392 (CONC 166716).

Pinus ponderosa Douglas ex Lawson "pino ponderosa" Arbol introducido en Chile, originario del oeste de Norteamérica. En Chile es cultivado con fines forestales. Esta especie ha sido introducida en la cuenca del Baker. Exs.: Sector Lago Chacabuco. E. Teneb 393 (CONC 166717).

\section{Podocarpaceae}

Lepidothamnus fonkii Phil. "ciprés enano" Subarbusto nativo de Chile y Argentina. Se distribuye entre las regiones de Los Lagos y Magallanes. Especie frecuente en el sur de la cuenca del Baker y abundante en toda la del Pascua. Estado de conservación: Vulnerable (Walters \& Guillett 1998). Exs.: Tortel, parte alta, en una turbera. R.Rodríguez y E. Teneb 4076 (CONC 166563). 


\section{Podocarpus nubigena Lindl.}

"mañio, mañilahuán” Arbol nativo de Chile y Argentina. Se encuentra entre las regiones de la Araucanía y la de Magallanes. Este árbol es frecuente al sur de la cuenca del Baker y en el río Pascua. Estado de conservación: Vulnerable (Walter \& Gillett 1998). Exs.: Río Baker. El Saltón. R. Rodríguez y E. Teneb 4280 (CONC 166634).

\section{MAGNOLIOPHYTA - MAGNOLIOPSIDA}

\section{Apiaceae \\ Azorella monantha Clos \\ Hierba perenne, crece en forma de cojines compactos. Nativa de Chile y Argentina. Se distribuye entre la Región Metropolitana y la de Magallanes. Es escasa en la cuenca del Baker. Exs.: Arenal en el río Baker, Blas Baker. E. Teneb 414 (CONC 166420).}

Azorella trifurcata (Gaertn.) Pers.

"leña de piedra" Hierba perenne, crece en forma de cojines compactos, nativa de Chile y Argentina. Se distribuye entre la Región del Bío-Bío y la de Magallanes. Es escasa en la cuenca del Baker. Exs.: Cochrane. Valle Chacabuco. C. Ramírez y O. Vidal s/n (CONC 166421).

Bolax caespitosa Hombr. et Jacquinot "llareta" Hierba perenne, crece en forma de cojines compactos, nativa de Chile, cosmopolita. Se distribuye entre las regiones de Los Lagos y la de Magallanes. Escasa en el Pascua. Exs.: Río Pascua. Pampa Quiroz. E. Teneb y A. Jiménez 4249 (CONC 166435).

\section{Conium maculatum $\mathrm{L}$.}

"cicuta"

Hierba anual, introducida en Chile, cosmopolita, originaria de europa. Se encuentra entre las regiones de Coquimbo a la de Magallanes. Es frecuente en la orilla de los caminos en el sector norte de la cuenca del Baker. Exs.: Cochrane. Sector Valle Castillo. R. Rodríguez y E. Teneb 4223 (CONC 166473).

Hydrocotyle chamaemorus Cham. et Schltdlt. Hierba perenne, nativa de Chile y Argentina. Se distribuye entre las regiones del Bío-Bío y la de Magallanes. Frecuente en la cuenca del Baker. Exs.: Cochrane. Lago Vargas. A. Jiménez 31 (CONC 166544).

Hydrocotyle indecora DC.

Hierba perenne, nativa de Chile y Argentina. En Chile se distribuye en la Región de Coquimbo y entre las regiones del Maule y de Los Lagos. Poco frecuente en la cuenca del río Pascua. Exs.: Río Bravo. E. Teneb 469 (CONC 166715).

Hydrocotyle poeppigii DC. "defecono" Hierba perenne, endémica en Chile. En Chile se distribuye entre las regiones del Bío-Bío y Aisén. Es poco frecuente en la cuenca del río Baker. Primera cita de la especie para la Región de Aisén. Exs.: Cochrane. Sector Lago Vargas. R. Rodríguez y E. Ruiz 4539 (CONC 166545).

Mulinum spinosum (Cav.) Pers. "neneo" Arbusto nativo de Chile y Argentina. En Chile se distribuye desde la Región de Atacama hasta la de Magallanes. Dominante en sectores áridos, al norte de la cuenca del Baker. Exs.: Cochrane. Sector El Manzano, curva Las Eses. R. Rodríguez y E. Ruiz 4301 (CONC 166595).

Osmorhiza berteroi DC.

"nukín" Hierba perenne, nativa en Chile y Argentina. En Chile se distribuye entre la Región de Los Lagos y la de Magallanes. Frecuente en el sotobosque de Nothofagus de la cuenca del Baker, menos frecuente en la del Pascua. Exs.: Cochrane. Sector Puerto Bertrand. R. Rodríguez y E. Ruiz 4355 (CONC 166611).

Schizeilema ranunculus (D'Urv.) Domin

Hierba perenne, nativa de Chile y Argentina. En Chile se distribuye entre la Región de Los Lagos y la de Magallanes. Es una especie frecuente en terrenos húmedos de la cuenca del río Baker. Exs.: Cochrane. Los Nadis. A. Jiménez 172 (CONC 166667).

Araliaceae

Pseudopanax laetevirens (Gay) Franchet "sauquillo" Arbol, nativo de Chile y Argentina. Se distribuye entre las regiones del Maule y Magallanes. Es un componente frecuente del estrato arbustivo de los bosques de ambas cuencas. Exs.: Entre Lago Leal y Quetru. R. Rodríguez y E. Teneb 4164 (CONC 166644).

\section{Asteraceae}

Abrotanella emarginata (Cass. ex Gaudich.) Cass. Hierba perenne, nativa de Chile y Argentina. En Chile crece en las regiones de Aisén y Magallanes. Es frecuente en suelos turbosos por sobre el límite arbóreo de la cuenca del río Pascua. Este es el primer registro de 
Plantas vasculares de los ríos Baker y Pascua: Rodríguez, R. eT AL.

la especie para la Región de Aisén. Exs.: Sector Pascua. C. Ramírez y O. Vidal s/n (CONC 166387).

Achillea millefolium $\mathrm{L}$ "mil en rama" Hierba perenne, introducida en Chile, originaria de Europa. Es posible encontrarla entre las regiones del Bío-Bío y Magallanes. Es una planta abundante a orillas de los caminos y en las praderas húmedas. Exs.: Cochrane. Pasarela El Manzano. R. Rodríguez y E. Ruiz 4321 (CONC 166394).

Adenocaulon chilense Less. "foiyelawén" Hierba perenne, nativa de Chile y Argentina. En Chile se distribuye entre las regiones de Valparaíso y Magallanes. Es una especie frecuente en los bosques de Nothofagus de la cuenca del Baker. Exs.: Cochrane. Sector Puerto Bertrand. R. Rodríguez y E. Ruiz 4348 (CONC 166395).

\section{Antennaria chilensis J.Remy}

Hierba perenne, nativa de Chile y Argentina. En Chile crece desde la Región de Coquimbo a la del Bío-Bío, y entre la Región de Aisén y la de Magallanes. Escasa en la cuenca del Baker. Exs.: Río Baker. Colonia Sur. E. Teneb 344 (CONC 166407).

Aster vahlii (Gaud.) Hook. et Arn.

Hierba anual, nativa de Chile y Argentina. En Chile se distribuye entre las regiones de O'Higgins y Magallanes. Es una especie frecuente en turberas de ambas cuencas. Exs.: Cochrane. Sector Puerto Bertrand. R. Rodríguez y E. Ruiz 4360 (CONC 166418).

\section{Baccharis magellanica (Lam.) Pers.}

"mosaiquillo"

Arbusto, forma céspedes, nativo de Chile y Argentina. En Chile se distribuye entre las regiones del Maule y Magallanes. Muy frecuente ambas cuencas. Exs.: Río Baker. El Saltón. R. Rodríguez y E. Teneb 4288 (CONC 166422).

Baccharis nivalis (Weed.) Sch.Bip. ex Phil.

Subarbusto nativo de Chile y Argentina. En Chile se encuentra entre las regiones del Bío-Bío y Magallanes. Es poco frecuente en arenales de las riberas de los ríos Baker y Pascua. Exs.: Camino de Cochrane a Tortel. Sector El Barrancoso. A. Marticorena 997 (CONC 166423).

Baccharis obovata Hook. et Arn. var.poeppigiana (DC.) Cabrera
Arbusto nativo de Chile y Argentina. En Chile se distribuye entre las regiones de Valparaíso y Aisén. Arbusto frecuente en ambas cuencas. Exs.: Camino de Cochrane a Bertrand, Ladera del cerro Tamango. R. Rodríguez y E. Teneb 4111 (CONC 166424).

Baccharis patagonica Hook. et Arn. "romerillo" Arbusto nativo en Chile y Argentina. Su distribución en Chile abarca desde la Región del Bío-Bío hasta Magallanes. Es un arbusto frecuente en la cuenca del río Baker. Exs.: Cochrane. Pasarela El Manzano. R. Rodríguez y E. Ruiz 4330 (CONC 166425).

Baccharis sagittalis (Less.) DC.

"kilafodu" Subarbusto nativo de Chile y Argentina. En Chile se distribuye entre las regiones de Tarapacá y Aisén. Es una especie poco frecuente en la cuenca del Baker. Exs.: Río Baker. E. Teneb 435 (CONC 166426).

\section{Carduus nutans L.}

"cardo"

Hierba anual, introducida en Chile, originaria de Europa. Se encuentra en las regiones de Valparaíso, Metropolitana, de la Araucanía y Aisén. Es una planta frecuente a orillas de camino en el sector de la cuenca del Baker. Este corresponde al primer registro de la especie en la Región de Aisén. Exs.: Cochrane. El Salto, Valle Grande. R. Rodríguez y E. Teneb 4214 (CONC 166452).

\section{Chiliotrichum diffusum (G.Forst.) Kuntze}

"fascine, romerillo"

Arbusto nativo de Chile y Argentina. En Chile se distribuye entre las regiones del Bío-Bío y la de Magallanes. Abundante en ambas cuencas. Exs.: Cochrane. Pasarela El Manzano. R. Rodríguez y E. Ruiz 4325 (CONC 166464).

\section{Chiliotrichum rosmarinifolium Less.}

"mata negra" Arbusto nativo de Chile y Argentina. En Chile se distribuye entre las regiones de O'Higgins y la de Magallanes. Es frecuente en la cuenca del Baker. Exs.: Río Baker. Angostura Tamango. E. Teneb 290 (CONC 166465).

Cirsium vulgare (Savi) Ten. "cardo" Hierba anual, introducida en Chile, originaria de Europa. Se encuentra entre las regiones de Valparaíso y Magallanes. Es una planta frecuente a orillas de camino en el sector de la cuenca del Baker. Exs.: 
Cochrane. El Salto, Valle Grande. R. Rodríguez y E. Teneb 4215 (CONC 166468).

\section{Conyza bonariensis (L.) Cronquist}

Hierba anual, introducida en Chile, originaria de América del Sur, presente también en Argentina. En Chile se encuentra entre las regiones de Tarapacá y la de Magallanes. Es una especie escasa en la cuenca del Baker. Exs.: Río Baker. Entre Nadis y Colonia. E. Teneb 206 (CONC 166474).

\section{Conyza floribunda Kunth}

Hierba anual, nativa en Chile y Argentina. En Chile se distribuye entre la Región de Coquimbo y la de Los Lagos. Poco frecuente en la cuenca del río Baker. Exs.: Cochrane. Puerto Bertrand. A. Jiménez 57 (CONC 166475).

Crepis capillaris (L.) Wallr.

Hierba anual, introducida en Chile, originaria de Europa. En Chile se encuentra entre las regiones de Valparaíso y Magallanes. Poco frecuente en la cuenca del Baker. Exs.: Río Baker. Angostura Tamango. E. Teneb 252 (CONC 166478).

Gamochaeta americana (Mill.) Wedd.

Hierba perenne, nativa de Chile y Argentina. Se distribuye entre las regiones de Valparaíso y Magallanes. Especie poco frecuente en la cuenca del Baker. Exs.: Sector Vagabundo. C. Ramírez y O. Vidal s/n (CONC 166516).

Gamochaeta spicata (Lam.) Cabrera

Hierba perenne, nativa de Chile y Argentina. En Chile se distribuye entre la Región de Valparaíso y Magallanes. Es una especies poco frecuente en el piso de los bosques de Nothofagus de ambas cuencas. Exs.: Río Pascua. E. Caviedes P3.11 (CONC 166517).

Hieracium chilense Less.

"kaltapul"

Hierba perenne, nativa en Chile y Argentina. Se distribuye entre las regiones del Maule y la de Magallanes. Especie frecuente en la cuenca del Baker. Exs.: Río Baker. Colonia Sur. E. Teneb 342 (CONC 166539).

Hieracium glaucifolium Poepp. ex Froel.

Hierba perenne nativa de Chile y Argentina. Se distribuye entre las regiones del Maule y Aisén. Escasa a orillas de los caminos en la cuenca del Baker.
Exs.: Confluencia de los ríos Nadis y Baker. E. Teneb 333 (CONC 166540).

Hypochaeris palustris (Phil.) De Wild.

Hierba perenne, nativa de Chile y Argentina. En Chile se distribuye desde la Región de la Araucanía a la de Magallanes. Especie frecuente en sitios húmedos de ambas cuencas. Exs.: Río Baker. Colonia Norte. E. Teneb 280 (CONC 166551).

Hypochaeris radicata L. "hierba del chancho" Hierba perenne, introducida en Chile, originaria de Europa. En Chile se encuentra desde la Región de Coquimbo a la de Magallanes. Abundante en sitios con pastoreo en la cuenca del Baker. Exs.: Cochrane, Colonia Norte. Sector El Carmen. R.Rodríguez y E. Teneb 4093 (CONC 166552).

\section{Lagenophora hariotii Franchet}

Hierba perenne, nativa en Chile y Argentina. Se distribuye desde la Región del Bío-Bío hasta la de Magallanes. Poco frecuente en turberas sobre el límite arbóreo de ambas cuencas. Exs.: Río Pascua. Angostura San Vicente. E. Teneb 361 (CONC 166559).

Leucheria achillaefolia Hook. et Arn.

Hierba perenne, nativa de Chile y Argentina. Se distribuye entre las regiones del Maule y la de Magallanes. Escasa en pastizales áridos de la zona norte de la cuenca del Baker. Exs.: Río Baker. Angostura Tamango. E. Teneb 248 (CONC 166564).

\section{Leucheria papillosa Cabrera}

Hierba perenne, nativa en Chile y Argentina. Se distribuye entre las regiones del Bío-Bío y Aisén. Escasa en la cuenca del Baker. Exs.: Cochrane. Nacimiento del Río Baker. C. Ramírez y O. Vidal s/n (CONC 166565).

\section{Macrachaenium gracile Hook.f.}

Hierba perenne, nativa en Chile y Argentina. Se distribuye entre las regiones de la Araucanía y Magallanes. Especie frecuente en bosques húmedos de las cuencas del Baker y Pascua. Exs.: Entre Vagabundo y Puerto Yungay. A. Marticorena 1069 (CONC 166579).

Madia sativa Molina

"madí, melosa"

Hierba anual, nativa en Chile y Argentina. Se distribuye entre la Región de Atacama y la de Magallanes. Escasa en sitios áridos, encontrada en 
la cuenca del Baker. Exs.: Cochrane. Sector Los Mellizos. A. Jiménez 191 (CONC 166580).

Mutisia spinosa Ruiz et Pav. "flor de la estrella" Arbusto trepador, nativo de Chile y Argentina. Se distribuye entre las regiones del Maule y la de Aisén. Es una enredadera frecuente en la cuenca del Baker. Exs.: Cochrane. Sector Puerto Bertrand. R. Rodríguez y E. Ruiz 4345 (CONC 166596).

Perezia lactucoides (Vahl) Less.

Hierba perenne, nativa de Chile y Argentina. En Chile se distribuyen entre las regiones de la Araucanía y la de Magallanes. Frecuente en sitios húmedos de la cuenca del Pascua. Exs.: Río Pascua. Angostura San Vicente. E. Teneb 358 (CONC 166613).

\section{Perezia linearis Less.}

Hierba perenne, nativa de Chile y Argentina. En Chile se distribuye entre las regiones del Bío-Bío y Magallanes. Poco frecuente en la cuenca del Baker. Exs.: Cochrane. Lago Esmeralda. C. Ramírez y O. Vidal $\mathrm{s} / \mathrm{n}(\mathrm{CONC} 166614)$

Perezia magellanica (L.f.) Less.

Hierba perenne, nativa de Chile y Argentina. En Chile se distribuye entre las regiones de Aisén y la de Magallanes. Frecuente en sitios húmedos por sobre el límite arbóreo de ambas cuencas. Exs.: Cochrane. Pasarela Ñadis. E. Teneb 55 (CONC 166615).

Perezia pedicularidifolia Less.

Hierba perenne, nativa de Chile y Argentina. Se distribuye entre las regiones del Bío-Bío y la de Magallanes. Escasa en la cuenca del Baker. Exs.: Río Baker. El Saltón. C. Ramírez y O. Vidal s/n (CONC 166616).

Perezia pilifera (D.Don) Hook. et Arn.

Hierba perenne, nativa de Chile y Argentina. Se distribuye entre las regiones de Valparaíso y la de Magallanes. Especie frecuente en sitios áridos de la cuenca del Baker. Exs.: Río Baker. Colonia Norte. E. Teneb 267 (CONC 166617).

Perezia recurvata (Vahl) Less.

Hierba perenne, nativa de Chile y Argentina. En Chile se encuentra entre las regiones de la Araucanía y la de Magallanes. Especie frecuente en sitios áridos de la cuenca del Baker. Exs.: Confluencia de los ríos Baker y Nadis. E. Teneb 234 (CONC 166618).
Senecio acanthifolius Hombr. et Jacquinot

Hierba perenne, nativa en Chile y Argentina. Se distribuye entre las regiones de Los Lagos y la de Magallanes. Es una especie frecuente en riachuelos y otros sitios húmedos de ambas cuencas. Exs.: Entre Vagabundo y Puerto Yungay. A. Marticorena 1081 (CONC 166672).

Senecio chrysocomoides Hook. et Arn.

Subarbusto nativo de Chile y Argentina. Sólo se encuentra en la Región de Aisén. Especie poco frecuente en la cuenca del Baker. Exs.: Cochrane. Sector Los Mellizos. A. Jiménez 175 (CONC 166673).

Senecio darwinii Hook. et Arn.

Subarbusto nativo de Chile y Argentina. Se distribuye entre las regiones de Los Lagos y la de Magallanes. Especie frecuente en la cuenca del Pascua. Exs.: Río Pascua. A. Jiménez 200 (CONC 166674).

Senecio leucomallus A.Gray var. incisus A.Gray Subarbusto nativo de Chile y Argentina. Se distribuye entre las regiones de Aisén y Magallanes. Poco frecuente en la cuenca del Baker. Primera referencia a esta especie en la Región de Aisén. Exs.: Cochrane. Sector Puerto Bertrand. R. Rodríguez y E. Ruiz 4357 (CONC 166675).

\section{Senecio neaei DC.}

Subarbusto nativo de Chile y Argentina. Se distribuye entre las regiones del Bío-Bío y Aisén. Poco frecuente en la cuenca del Baker. Exs.: Cochrane. Sector Valle Castillo. R. Rodríguez y E. Teneb 4224 (CONC 166676).

Senecio patagonicus Hook. et Arn.

Subarbusto nativo de Chile y Argentina. Crece en las regiones de Aisén y Magallanes. Frecuente en la cuenca del Baker. Exs.: Río Baker. Angostura Tamango. E. Teneb 244 (CONC 166677).

Senecio philippii Sch. Bip. ex Wedd.

Subarbusto, nativo de Chile y Argentina. Se distribuye entre las regiones de Los Lagos y Magallanes. Especie poco frecuente, crece sobre el límite arbóreo de las cuencas del Pascua y Baker. Río Vagabundo. Exs.: E. Teneb 459 (CONC 166714).

\section{Senecio skottsbergii Cabrera}

Subarbusto, nativo de Chile y Argentina. Se distribuye entre la Región de Aisén y la de Magallanes. Poco frecuente en la cuenca del Baker. 
Exs.: Cochrane. Río Nadis, arenales. E. Teneb 102 (CONC 166678).

Senecio smithii DC.

"hualtata"

Hierba perenne, nativa de Chile y Argentina. Se distribuye entre las regiones de la Araucanía y la de Magallanes. Frecuente en áreas muy húmedas de ambas cuencas. Exs.: Cochrane. Lago Vargas. E. Teneb 85 (CONC 166679).

Senecio trifurcatus (G.Forst.) Less. ex DC.

Hierba perenne, nativa de Chile y Argentina. Se distribuye entre las regiones de la Araucanía y la de Magallanes. Frecuente en suelos turbosos de ambas cuencas. Exs.: Cochrane. Pasado Los Nadis hacia el sur. A. Marticorena 1008 (CONC 166680).

\section{Senecio tríodon Phil.}

Subarbusto nativo de Chile y Argentina. Se distribuye entre las regiones de Maule y Aisén. Poco frecuente en la cuenca del Baker. Exs.: Cochrane. Puerto Bertrand. C. Ramírez y O. Vidal s/n (CONC 166681).

Solidago chilensis Meyen "fulel, huellén" Hierba perenne, nativa de Chile y Argentina. En Chile se distribuye entre las regiones de Atacama y Aisén. Es una planta frecuente en la cuenca del Baker. Exs.: Cochrane. Sector Valle Castillo. R. Rodríguez y E. Teneb 4222 (CONC 166685).

Sonchus oleraceus L. "cerrajilla, ñilhue" Hierba anual, introducida en Chile, originaria de Europa. Su distribución en Chile es desde la Región de Atacama hasta la de Magallanes. Poco frecuente en la orilla de los caminos en la cuenca del Baker (C.Ramírez *).

Tanacetum vulgare L. " "plumilla, ajenjo chileno" Hierba perenne, introducida en Chile, originaria de Europa. En Chile se encuentra desde la Región del Bío-Bío hasta Magallanes. Planta medicinal, por lo que se cultiva en algunos lugares de la cuenca del Pascua. Exs.: Río Bravo. E. Teneb 468 (CONC 166713).

\section{Taraxacum officinale Weber ex F.H.Wigg}

"diente de león"

Hierba perenne, introducida en Chile, originaria de Europa. En Chile se encuentra entre las regiones de Antofagasta y Magallanes. En una planta abundante en áreas bajo presión ganadera de la cuenca del Baker. Exs.: Cochrane. Sector El Manzano, curva Las Eses. R. Rodríguez y E. Ruiz 4305 (CONC 166687).

\section{BERBERIDACEAE}

Berberis darwinii Hook.

"michay"

Arbusto nativo de Chile y Argentina. Se distribuye entre la Región del Maule y Aisén. Es abundante en la cuenca del Baker y menos frecuente en la del Pascua. Exs.: Río Baker. El Saltón. R. Rodríguez y E. Teneb 4285 (CONC 166428).

\section{Berberis empetrifolia Lam.}

"zarcilla, calafate enano"

Arbusto pequeño, nativo de Chile y Argentina. Se encuentra entre las regiones de Coquimbo y Magallanes. Es frecuente en zonas áridas de la cuenca del Baker. Exs.: Cochrane. Pasarela El Manzano. R. Rodríguez y E. Ruiz 4319 (CONC 166429).

\section{Berberis ilicifolia L.f.}

"chelia, michay" Arbusto nativo de Chile y Argentina. Se distribuye entre las regiones de Los Lagos y de Magallanes. Poco frecuente en la cuenca del Baker pero abundante en la del Pascua. Exs.: Río Pascua. Pampa Quiroz. E. Teneb y A. Jiménez 4233 (CONC 166430).

Berberis microphylla G.Forst

"calafate" Arbusto nativo de Chile y Argentina. Se distribuye entre las regiones de O'Higgins y Magallanes. Frecuente en ambas cuencas, su fruto es una baya comestible. Exs.: Río Baker. El Saltón. R. Rodríguez y E. Teneb 4284 (CONC 166431).

BignONiaceAe

Campsidium valdivianum (Phil.) Skottsb. "pilpilvoqui"

Arbusto trepador, nativo de Chile y Argentina. Se distribuye entre las regiones del Bío-Bío y de Magallanes. Frecuente en los bosques siempreverdes de la cuenca del Pascua. Exs.: Río Pascua. Angostura San Vicente. R. Rodríguez y E. Teneb 4189 (CONC 166449).

\section{BoraginaCEAE}

Myosotis stricta Link ex Roem. et Schult. Hierba anual, introducida en Chile, origen euroasiático. Se encuentra en las regiones de Aisén y Magallanes. Frecuente en la cuenca del Baker. Exs.: Cochrane. Sector El Manzano, curva Las Eses. R. Rodríguez y E. Ruiz 4295 (CONC 166598).

\section{Brassica rapa $\mathrm{L}$.}

BRASSICACEAE

Hierba anual, introducida en Chil 
originaria del centro de Europa. Se encuentra entre las regiones de Antofagasta y Magallanes. Frecuente en la orilla de los caminos en la cuenca del Baker. Exs.: Cochrane. El Salto. R. Rodríguez y E. Ruiz 4459 (CONC 166436).

Capsella bursa-pastoris (L.) Medik.

"bolsita del pastor"

Hierba anual, introducida en Chile, originaria de Europa mediterránea. Se encuentra en todo el territorio nacional (Atacama a Magallanes). Frecuente en la orilla de los caminos en la cuenca del Baker (C.Ramírez *).

Cardamine bonariensis A.L.Juss. ex Pers.

Hierba perenne, nativa de Chile y Argentina. En Chile se distribuye entre las regiones de Valparaíso y de Magallanes. Poco frecuente en la cuenca del Baker. Exs.: Cochrane. Sector Los Nadis. C. Ramírez O. Vidal $\mathrm{s} / \mathrm{n}$ (CONC 166450).

Cardamine glacialis (G.Forst.) DC.

Hierba perenne nativa de Chile y Argentina. Presenta una distribución disjunta, se encuentra desde la región de Atacama a la de Magallanes. Poco frecuente en bosques de la cuenca del Baker. Exs.: Río Baker. Colonia Norte. E. Teneb 274 (CONC 166451).

\section{Draba magellanica Lam.}

Hierba perenne, nativa en Chile y Argentina. Se distribuye entre las regiones de Aisén y de Magallanes. Poco frecuente en la cuenca del Baker. Exs.: Cochrane. Lago Esmeralda. C. Ramírez y O. Vidal s/n (CONC 166488).

\section{Calceolariaceae}

Calceolaria biflora Lam. "topa-topa, zapatito" Hierba anual, nativa de Chile y Argentina. Se distribuye entre las regiones de Aisén y Magallanes. Frecuente en la cuenca del Baker. Exs.: Cochrane, Colonia Norte. Sector El Carmen. R. Rodríguez y E. Teneb 4109 (CONC 166443).

Calceolaria tenella Poepp.

Hierba perenne, nativa de Chile y Argentina. Se distribuye entre las regiones de Los Lagos y de Magallanes. Frecuente en la cuenca del Baker. Exs.: Camino de Cochrane a Tortel. Puente Nadi. R. Rodríguez y E. Teneb 4129 (CONC 166444).
Callitrichaceae

Callitriche lechleri (Hegelm.) Fassett

"huenchecó"

Hierba anual, acuática arraigada, nativa en Chile y Argentina. Se distribuye entre las regiones de la Araucanía y de Magallanes. Poco frecuente en la cuenca del Baker. Exs.: Tortel. Desembocadura del río Baker. R. Rodríguez y E. Teneb 4198 (CONC 166445).

\section{Campanulaceae}

Downingia pusilla (Poepp. ex A.DC.) Torr.

Hierba anual, nativa en Chile y Argentina. Se distribuye entre las regiones de Valparaíso y Aisén. Frecuente en la cuenca del Baker. Primera cita de esta especie para la Región de Aisén. Exs.: Cochrane. Sector El Manzano. A. Marticorena 931 (CONC 166487).

Lobelia oligophylla (Wedd.) Lammers

Hierba perenne, nativa de Chile y Argentina. Se distribuye a lo largo de todo Chile (Tarapacá a Magallanes). Es poco frecuente en la cuenca del Baker. Exs.: Cochrane. Pasado Los Nadis hacia el sur. A. Marticorena 1009 (CONC 166568).

\section{Caryophyllaceae}

Colobanthus quitensis (Kunth) Bartl.

Hierba perenne, nativa en Chile y Argentina. Se distribuye en todo Chile (Atacama a Magallanes). Hierba escasa en arenales de la cuenca del Baker. Exs.: Cochrane. Valle Chacabuco. C. Ramírez y O. Vidal s/n (CONC 166472).

\section{Arenaria serpens Kunth}

Hierba perenne, nativa de Chile y Argentina. Se distribuye en Chile continental (Atacama a Magallanes). Frecuente en arenales húmedos de la cuenca del Baker. Exs.: Cochrane. Sector Puerto Bertrand. R. Rodríguez y E. Ruiz 4359 (CONC 166412).

\section{Arenaria serpyllifolia $\mathrm{L}$.}

Hierba perenne, introducida en Chile, originaria de Europa. Se encuentra entre las regiones de Coquimbo y de Magallanes. Es frecuente en la orilla de los caminos en la cuenca del Baker. Exs.: Río Baker. Angostura Tamango. E. Teneb 251 (CONC 166413).

Cerastium arvense $\mathrm{L}$. "cuernecita" Hierba perenne, introducida en Chile, originaria de 
Europa, cosmopolita. Se encuentra desde la Región de Coquimbo hasta Magallanes. Frecuente en sitios bajo presión ganadera de la cuenca del Baker. Exs.: Cochrane. Sector El Manzano, curva Las Eses. R. Rodríguez y E. Ruiz 4294 (CONC 166461).

\section{Cerastium glomeratum Thuill.}

Hierba anual, introducida en Chile, originaria de Europa, cosmopolita. Se encuentra desde la Región de Coquimbo hasta Magallanes. Es frecuente en sitios bajo presión ganadera en la cuenca del Baker. Exs.: Lago Juncal. Sector Poco a Poco. R. Rodríguez y E. Teneb 4261 (CONC 166462).

\section{Sagina procumbens $\mathrm{L}$.}

Hierba perenne, introducida en Chile, cosmopolita. Se encuentra entre las regiones de la Araucanía y de Magallanes. Frecuente en la orilla de los caminos de ambas cuencas. Exs.: Cochrane. Puente Barrancoso. R. Rodríguez y E. Ruiz 4416 (CONC 166662).

Silene magellanica (Desr.) Bocquet

Hierba perenne, nativa en Chile y Argentina. Se distribuye en las regiones de Aisén y Magallanes. Frecuente en la cuenca del Baker. Primera cita de esta especie para la Región de Aisén. Exs.: Cochrane. Sector Los Mellizos. A. Jiménez 178 (CONC 166683).

\section{Celastraceae}

Maytenus boaria Molina "maitén" Arbol nativo de Chile y Argentina. Su distribución va desde la Región de Atacama a la de Magallanes (Dollenz 2003). Restringido al sector Los Maitenes, cerca de Cochrane, en la cuenca del Baker. Es el primer registro de esta especie para la Región de Aisén. Exs.: Cochrane. Sector Valle Castillo. R. Rodríguez y E. Teneb 4227 (CONC 166582).

\section{Maytenus disticha (Hook.f.) Urb.}

Arbusto nativo de Chile y Argentina. Se distribuye entre las regiones del Maule y de Magallanes. Frecuente en los bosques de lenga y en algunos bosques mixtos sin intervención de ambas cuencas. Exs.: Río Colonia. Sector La Península. R. Rodríguez y E. Teneb 4251 (CONC 166583).

\section{Maytenus magellanica (Lam.) Hook.f.}

"leña dura" Arbol nativo en Chile y Argentina. Se distribuye entre las regiones del Maule y de Magallanes. Componente frecuente de los bosques siempreverdes de ambas cuencas. Exs.: Cochrane. Lago Largo. R. Rodríguez y E. Ruiz 4497 (CONC 166584).

\section{Cornaceae}

Griselinia ruscifolia (Clos) Ball "lilinquén" Arbusto trepador, nativo de Chile y Argentina. Se distribuye entre las regiones de Los Lagos y de Magallanes. Frecuente en bosques siempreverdes de ambas cuencas. Exs.: Río Pascua. Angostura San Vicente. R. Rodríguez y E. Teneb 4197 (CONC 166534).

\section{Crassulaceae}

Sedum acre $\mathrm{L}$

Hierba perenne, introducida en Chile, originaria de Europa, Asia y Africa. Se encuentra desde la Región de la Araucanía hasta la de Magallanes. Es escasa en sitios áridos y arenales de la cuenca del río Baker. Exs.: Cochrane. Pasarela El Manzano. R. Rodríguez y E. Ruiz 4307 (CONC 166671).

\section{Cunoniaceae}

Weinmannia trichosperma Cav.

"tineo" Arbol nativo de Chile y Argentina. Se distribuye desde la Región del Maule hasta la de Magallanes. Es una especie frecuente en la parte sur de la cuenca del Baker y en la cuenca del Pascua. Exs.: Río Baker. El Saltón. R. Rodríguez y E. Teneb 4293 (CONC 166707).

Desfontainia fulgens D.Don

"taique"

Arbusto con hojas punsantes, nativo de Chile y Argentina. Se distribuye entre las regiones del Maule y de Magallanes. Frecuente en los bosques siempreverdes de la cuenca del Baker y abundante en la del Pascua. Exs.: Tortel, parte alta, en una turbera. R. Rodríguez y E. Teneb 4080 (CONC 166484).

\section{DONATIACEAE}

Donatia fascicularis J.R.Forst. et G.Forst.

Hierba perenne, nativa de Chile y Argentina. Se distribuye desde la Región del Bío-Bío hasta la de Magallanes. Es un elemento dominante en las turberas duriherbosas (pulvinadas) de ambas cuencas. Exs.: Tortel, parte alta, en una turbera. R. Rodríguez y E. Teneb 4084 (CONC 166486).

\section{Drosera uniflora Willd. "atrapa moscas"} Hierba perenne, insectívora, de vistoso color rojo; nativa en Chile y Argentina. Se distribuye desde la 
Región de la Araucanía hasta la de Magallanes. Frecuente en tuberas duriherbosas de ambas cuencas. Exs.: Tortel, parte alta, en una turbera. R. Rodríguez y E. Teneb 4082 (CONC 166491).

\section{EMPETRACEAE}

Empetrum rubrum Vahl ex Willd.

"mulwén, brecillo"

Arbusto decumbente, nativo en Chile y Argentina. Se distribuye entre las regiones de Valparaíso y de Magallanes. Es frecuente en bosques, turberas y coironales de ambas cuencas. Exs.: Río Baker. El Saltón. R. Rodríguez y E. Teneb 4281 (CONC 166497).

\section{EPACRIDACEAE}

Lebetanthus myrsinites (Lam.) Dusén

"chaurilla"

Arbusto escandente, nativo en Chile y Argentina. Se distribuye en las regiones de Aisén y Magallanes. Muy frecuente en los bosques siempreverdes de la cuenca del Pascua. Exs.: Río Pascua. Angostura San Vicente. R. Rodríguez y E. Teneb 4190 (CONC 166562).

\section{ERICACEAE}

Gaultheria antarctica Hook.f.

Subarbusto nativo de Chile y Argentina. Se distribuye entre las regiones de la Araucanía y de Magallanes. Es poco frecuente en ambas cuencas. Exs.: Río Pascua. Pampa Quiroz. E. Teneb y A. Jiménez 4243 (CONC 166518).

Gaultheria mucronata (L.f.) Hook et Arn.

"chaura, chique"

Arbusto de hojas punzantes, nativo de Chile y Argentina. Se distribuye entre las regiones del BíoBío y de Magallanes. Es muy frecuente en ambas cuencas. Exs.: Cochrane. Pasarela El Manzano. R. Rodríguez y E. Ruiz 4326 (CONC 166519).

Gaultheria phillyreifolia (Pers.) Sleumer "chaura"

Arbusto de hojas punzantes, nativo de Chile y Argentina. Se distribuye entre las regiones de Coquimbo y de Magallanes. Poco frecuente en la cuenca del Baker. Exs.: Río Baker. El Saltón. R. Rodríguez y E. Teneb 4286 (CONC 166520).

Gaultheria poeppigii DC.

Subarbusto nativo en Chile y Argentina. Se distribuye entre las regiones del Maule y de Aisén. Poco frecuente en la cuenca del Baker. Exs.: Cuesta Barrancoso, parte media. R. Rodríguez y E. Ruiz 4395 (CONC 166521).

Gaultheria pumila (L.f.) D.J.Middleton

Arbusto nativo de Chile y Argentina. Se distribuyen entre las regiones Metropolitana y de Magallanes. Poco frecuente en ambas cuencas. Exs.: Cochrane. Puente río El Maitén. R. Rodríguez y E. Ruiz 4337 (CONC 166522).

\section{Escalloniaceae}

Escallonia alpina Poepp. ex DC.

Arbusto nativo de Chile y Argentina. Se distribuye entre las regiones de Valparaíso y de Magallanes. Poco frecuente en sitios húmedos de ambas cuencas. Exs.: Cochrane. Los Nadis. A. Jiménez 82 (CONC 166500).

Escallonia rosea Griseb. "siete camisas" Arbusto nativo de Chile y Argentina. Se distribuye entre las regiones del Maule y de Magallanes. Poco frecuente en ambas cuencas (Baker y Pascua). Exs.: Cuesta Barrancoso, parte media. R. Rodríguez y E. Ruiz 4400 (CONC 166501).

Escallonia rubra (Ruiz et Pav.) Pers.

"ñipa, müki"

Arbusto nativo de Chile y Argentina. Se distribuye entre las regiones de Valparaíso y de Magallanes. Frecuente en ambas cuencas. Exs.: Camino de Cochrane a Bertrand, Angostura El Manzano. R. Rodríguez y E. Teneb 4120 (CONC 166502).

\section{Escallonia serrata $\mathrm{Sm}$.}

Arbusto nativo de Chile y Argentina. Su distribución va desde la Región de Los Lagos hasta la de Magallanes. Es frecuente en la cuenca del Pascua. Exs.: Río Pascua. Angostura San Vicente. E. Teneb 368 (CONC 166503).

Escallonia virgata (Ruiz et Pav.) Pers. "mata negra, meki" Arbusto nativo de Chile y Argentina. Se distribuye entre las regiones del Maule y de Magallanes. Frecuente en sectores húmedos de la cuenca del Baker. Exs.: Cochrane. Puente río El Maitén. R. Rodríguez y E. Ruiz 4334 (CONC 166504).

Tribeles australis Phil.

Hierba perenne, nativa de Chile y Argentina. Se distribuye entre las regiones de Los Lagos y de 
Magallanes. Frecuente en turberas de ambas cuencas. Exs.: Cochrane. Lago Vargas. E. Teneb 81 (CONC 166690).

EUPHORBIACEAE

Dysopsis glechomoides (A.Rich.) Müll.-Arg. "pihuichén-domo"

Hierba perenne, nativa de Chile. Presenta una distribución disjunta, se encuentra en P.N. Fray Jorge en la Región de Coquimbo y desde la Región de Los Lagos hasta la de Magallanes. Frecuente en bosques poco intervenidos de las cuencas del Baker y Pascua. Exs.: Río Baker. Colonia Norte. E. Teneb 273 (CONC 166492).

\section{FABACEAE}

\section{Adesmia corymbosa Clos}

Hierba perenne, nativa de Chile y Argentina. Se distribuye entre las regiones de Valparaíso y de Aisén. Escasa en la cuenca del Baker. Exs.: Río Baker. Angostura Tamango. E. Teneb 288 (CONC 166396).

Laburnum anagyroides Medik. "lluvia de oro" Arbusto, introducido en Chile, originario de Europa, cosmopolita. En Chile es cultivado con fines ornamentales, especialmente en las calles de Cochrane. Exs.: Cochrane. Puente Nadis. R. Rodríguez y E. Ruiz 4502 (CONC 166558).

\section{Lathyrus magellanicus Lam.}

"arvejilla"

Hierba perenne, trepadora, nativa de Chile y Argentina. Se distribuye entre las regiones de Coquimbo y de Magallanes. Frecuente en los matorrales bajos de la cuenca del Baker. Exs.: Cochrane. Pasarela El Manzano. R. Rodríguez y E. Ruiz 4311 (CONC 166561).

\section{Lupinus polyphyllus Lindl.}

Hierba perenne, introducida en Chile, originaria de Norteamérica occidental. En Chile ha sido recientemente reportada para la Región de Aisén. Abundante en la orilla de los caminos en la cuenca Baker. Planta de uso ornamental y naturalizada en el sur de Chile. Exs.: Río Baker. Frente a la confluencia con el río Neff. R. Rodríguez y E. Teneb 4545 (CONC 166571).

Trifolium dubium Sibth.

"trebillo" Hierba anual, introducida en Chile, originaria de Europa. Se encuentra entre las regiones de Valparaíso y de Magallanes. Es una hierba palatable frecuente en praderas secundarias de la cuenca del Baker. Exs.:
Cuesta Barrancoso, parte media. R. Rodríguez y E. Ruiz 4394 (CONC 166691).

Trifolium pratense $\mathrm{L}$. "trébol rosado" Hierba perenne, introducida en Chile, originaria de Europa. Se encuentra desde las regiones de Valparaíso y la de Magallanes. Es una hierba palatable frecuente en praderas secundarias de la cuenca del Baker (C.Ramírez *).

\section{Trifolium repens $\mathrm{L}$.}

"trébol blanco" Hierba perenne, introducida en Chile, originaria de Europa. Se encuentra desde la Región de Coquimbo hasta la de Magallanes. Frecuente en pastizales secundarios de la cuenca del Baker (C.Ramírez *).

Vicia nigricans Hook. et Arn. "arvejilla" Hierba perenne, trepadora, nativa en Chile y Argentina. Se distribuye entre las regiones de Valparaíso y de Aisén. Frecuente entre matorrales de la cuenca del Baker. Primera cita de esta especie en la Región de Aisén. Exs.: Cochrane. Sector Puerto Bertrand. R. Rodríguez y E. Ruiz 4343 (CONC 166700).

Vicia tetrasperma (L.) Schreb.

Hierba perenne, trepadora, introducida en Chile, originaria de Europa. Se encuentra entre las regiones de Atacama y la de Magallanes. Escasa entre matorrales de la cuenca del Baker. Exs.: Camino de Cochrane a Tortel. Sector El Barrancoso. A. Marticorena 999 (CONC 166701).

\section{Flacourtiaceae}

Azara lanceolata Hook.f. "aromo" Arbusto, nativo de Chile y Argentina. Se distribuye entre las regiones del Bío-Bío y de Aisén. Es un elemento leñoso frecuente en los bosques siempreverdes de la cuenca del Pascua. Exs.: Entre Lago Leal y Quetru. R. Rodríguez y E. Teneb 4167 (CONC 166419).

\section{Gentianaceae}

Centaurium littorale (Turner) Gilmour

Hierba perenne, introducida en Chile, originaria de Europa, cosmopolita. En Chile se encuentra entre la Región de Los Lagos y de Aisén. Es una planta frecuente en sitios áridos y sujetos a presión ganadera de la cuenca del Baker. Este es el primer registro de la especie para la Región de Aisén. Exs.: Cochrane. Sector Los Mellizos. A. Jiménez 142 (CONC 166460). 


\section{Geraniaceae \\ Erodium cicutarium (L.) L'Hérit. ex Aiton}

"alfilerillo"

Hierba anual, introducida en Chile, originaria de la región mediterránea de Europa, cosmopolita. Se encuentra en todo Chile. Es frecuente en sitios áridos y bancos arenosos de la cuenca del Baker. Exs.: Cochrane. Sector El Manzano, curva Las Eses. R. Rodríguez y E. Ruiz 4299 (CONC 166499).

\section{Geranium berteroanum Colla}

Hierba perenne, nativa en Chile y Argentina. Se encuentra en la Región Metropolitana, del Bío-Bío y de Magallanes. Es una planta frecuente en la cuenca del río Baker. Exs.: Cochrane. El Salto, Valle Grande. R. Rodríguez y E. Teneb 4210 (CONC 166527).

\section{Geranium sessiliflorum Cav.}

Hierba perenne, nativa de Chile y Argentina. Se distribuye entre la Región Metropolitana y la de Magallanes. Es un planta frecuente en la cuenca del Baker. Exs.: Cochrane. Lago Esmeralda. C. Ramírez y O. Vidal s/n (CONC 166528).

\section{GESNERIACEAE}

Asteranthera ovata (Cav.) Hanst. "estrellita" Arbusto nativo de Chile y Argentina. Se distribuye entre las regiones del Bío-Bío y de Magallanes. Poco frecuente en la cuenca del Baker (C.Ramírez *).

Mitraria coccinea Cav.

"botellita"

Arbusto escandente con llamativas flores rojas, nativo de Chile y Argentina. Se distribuye entre las regiones del Bío-Bío y de Magallanes. Frecuente en bosques siempreverde de la cuenca del Baker y abundante en la del Pascua. Exs.: Entre Lago Leal y Quetru. R. Rodríguez y E. Teneb 4169 (CONC 166593).

\section{Grossulariaceae}

Ribes cucullatum Hook. et Arn.

Arbusto nativo de Chile y Argentina. Se distribuye desde la Región de Valparaíso a la de Magallanes. Frecuente en la cuenca del Baker. Exs.: Cochrane. Sector El Manzano, curva Las Eses. R. Rodríguez y E. Ruiz 4303 (CONC 166651).

Ribes magellanicum Poir. "parrilla" Arbusto nativo de Chile y Argentina. Se distribuye desde la Región de O’Higgins hasta la de Magallanes. Frecuente en ambas cuencas. Exs.: Río Pascua.
Angostura San Vicente. R. Rodríguez y E. Teneb 4194 (CONC 166652).

Ribes trilobum Meyen "parrilla" Arbusto endémico en Chile. Se distribuye entre la Región de Coquimbo y la de Aisén. Escaso en bosques caducifolios de la cuenca del Baker. Primera cita de la especie para la Región de Aisén. Exs.: Cochrane. El Salto. R. Rodríguez y E. Ruiz 4460 (CONC 166653).

\section{GunNERACEAE}

\section{Gunnera lobata Hook.f.}

Hierba perenne, nativa de Chile y Argentina. Se encuentra entre la Región de Los Lagos y la de Magallanes. Poco frecuente en turberas de la cuenca del Pascua. Exs.: Balboa. E. Teneb 193 (CONC 166535).

\section{Gunnera magellanica Lam.}

Hierba perenne, nativa de Chile y Argentina. Se distribuye entre las regiones Metropolitana y de Magallanes. Frecuente en pequeños cursos de agua y sitios inundados de ambas cuencas. Exs.: Tortel, parte alta, en una turbera. R.Rodríguez y E. Teneb 4081 (CONC 166536).

Gunnera tinctoria (Molina) Mirb. "pangue" Hierba perenne de gran tamaño, nativa de Chile y Argentina. Se distribuye entre las regiones de Coquimbo y de Magallanes. Frecuente en la cuenca del Baker. Exs.: Cochrane. Puente Nadis. R. Rodríguez y E. Ruiz 4501 (CONC 166537).

\section{Haloragaceae \\ Myriophyllum quitense Kunth}

"hierba del sapo" Hierba perenne, acuática, nativa de Chile y Argentina. Se distribuye entre las regiones de Antofagasta y de Magallanes. Escasa en lagunas y charcas de la cuenca del Baker. Exs.: Cochrane. Colonia Sur. R. Rodríguez y E. Ruiz 4473 (CONC 166599).

\section{Hippuris vulgaris L. "pinito de agua"} Hierba perenne, acuática, nativa de Chile y Argentina. Se distribuye desde la Región de Los Lagos hasta la de Magallanes. Escasa en charcas y lagunas de la cuenca del Baker. Exs.: Cochrane. Colonia Sur. R. Rodríguez y E. Ruiz 4475 (CONC 166541). 


\section{HYDROPHYLLACEAE}

Phacelia secunda J.F.Gmel. "cuncuna" Hierba perenne, nativa de Chile y Argentina. Se encuentra desde la Región de Tarapacá a la de Magallanes. Es frecuente en sitios alterados de la cuenca del Baker. Exs.: El Maitén. Sector río Neff. Rodríguez y E. Teneb 4275 (CONC 166619).

\section{LAMiACEAE \\ Mentha piperita $\mathrm{L}$. \\ "menta"}

Hierba perenne, introducida en Chile, originaria de Europa. Se encuentra entre las regiones de Antofagasta y de Aisén. Es cultivada con fines medicinales en la cuenca del Baker. Exs.: Cochrane. El Salto, Valle Grande. R. Rodríguez y E. Teneb 4211 (CONC 166585).

Prunella vulgaris L.

"hierba mora" Hierba perenne, introducida en Chile, originaria de Europa. Se encuentra entre las regiones de Coquimbo y de Magallanes. Frecuente en la orilla de los caminos de la cuenca del Baker. Exs.: El Maitén. Sector río Neff. Rodríguez y E. Teneb 4267 (CONC 166643).

\section{LENTIBULARIACEAE}

Pinguicula antarctica Vahl

Hierba perenne, nativa de Chile y Argentina. Se distribuye entre las regiones de la Araucanía y de Magallanes. Escasa en turberas por sobre el límite arbóreo de la cuenca del Pascua. Exs.: Río Pascua. E. Teneb 162 (CONC 166624).

\section{Pinguicula chilensis Gay}

Hierba perenne, nativa de Chile y Argentina. Se distribuye entre la Región del Maule y de Aisén. Escasa, encontrada en turberas en el límite arbóreo de la cuenca del Baker. Este es el primer registro de la especie para la Región. Exs.: Cochrane. Puerto Bertrand. C. Ramírez y O. Vidal s/n (CONC 166625).

\section{Loasa bergii Hieron.}

\section{LOASACEAE}

Hierba perenne, nativa de Chile y Argentina. Se distribuye en las regiones de Aisén y Magallanes. Poco frecuente en la cuenca del Baker. Exs.: Cochrane. Sector Los Mellizos. A. Jiménez 190 (CONC 166567).

\section{Malvaceae}

Malva moschata $\mathrm{L}$.

Hierba perenne, introducida en Chile, originaria del sur de Europa y del norte de Africa. Se encuentra desde la Región de Los Lagos a la de Aisén. Escasa. Exs.: Lago Vargas. E. Teneb 375 (CONC 166712).

\section{MisodendRaceAe}

Misodendrum angulatum Phil.

"cabello de ángel"

Subarbusto parásito sobre Nothofagus, nativo de Chile y Argentina. Se distribuye entre las regiones de la Araucanía y de Aisén. Frecuente en la cuenca del Pascua. Exs.: Río Pascua. Pampa Quiroz. E. Teneb y A. Jiménez 4239 (CONC 166588).

Misodendrum gayanum Tiegh.

Subarbusto parásito sobre Nothofagus, nativo de Chile y Argentina. Se distribuye entre la Región del Bío-Bío y de Aisén. Escaso en la cuenca del Pascua. Exs.: Entre Vagabundo y Puerto Yungay. A. Marticorena 1076 (CONC 166589).

\section{Misodendrum linearifolium DC.}

"cabello de ángel"

Subarbusto parásito de Nothofagus, nativo de Chile y Argentina. Se distribuye entre las regiones del Maule y de Magallanes. Escaso en ambas cuencas. Exs.: Cochrane. Predio Pucchi. R, Rodríguez y E. Ruiz 4423 (CONC 166590).

Misodendrum punctulatum Banks ex DC. "injerto, cabello de árbol" Subarbusto parásito de Nothofagus. Nativo de Chile y Argentina. Se distribuye entre las regiones del Maule y de Magallanes. Frecuente en ambas cuencas. Exs.: Cochrane, Colonia Norte. Sector El Carmen. R. Rodríguez y E. Teneb 4092 (CONC 166591).

\section{Misodendrum quadriflorum DC.}

Subarbusto parásito en Nothofagus, nativo de Chile y Argentina. Se distribuye entre las regiones del BíoBío y de Magallanes. Escasa en ambas cuencas. Exs.: Río Baker. Colonia Norte. E. Teneb 283 (CONC 166592).

\section{Myrtaceae}

Amomyrtus luma (Molina) D.Legrand et Kausel "luma" Arbol nativo de Chile y Argentina. Se distribuye entre las regiones del Maule y la de Aisén. Poco frecuente en bosques siempreverdes de la cuenca del Baker. Exs.: Cruce Vagabundo. A. Marticorena 1029(CONC 166404). 


\section{Myrteola nummularia (Poir.) O.Berg}

"daudapo"

Subarbusto rastrero, nativo de Chile y Argentina. Se distribuye entre las regiones del Bío-Bío y de Magallanes. Frecuente en turberas de ambas cuencas. Exs.: Entre Lago Leal y Quetru. R. Rodríguez y E. Teneb 4156 (CONC 166600).

Tepualia stipularis (Hook. et Arn.) Griseb. "tepú" Arbusto nativo de Chile y Argentina. Se distribuye entre las regiones del Maule y de Magallanes. Frecuente en áreas turbosas cerca de Caleta Tortel y en toda la cuenca del Pascua. Exs.: Río Baker. El Saltón. R. Rodríguez y E. Teneb 4292 (CONC 166688).

\section{NothofagaceAe}

Nothofagus antarctica (G.Forst.) Oerst. "nirre" Arbol nativo de Chile y Argentina. Se distribuye entre las regiones del Maule y de Magallanes. Frecuente en variados ambientes de ambas cuencas. Exs.: Cochrane, Colonia Norte. Sector El Carmen. R. Rodríguez y E. Teneb 4096 (CONC 166604).

Nothofagus betuloides (Mirb.) Oerst. "coigüe de Magallanes" Arbol nativo de Chile y Argentina. Se encuentra entre las regiones de Los Lagos y de Magallanes. Frecuente en sitios turbosos de la cuenca del Baker y es dominante en los bosques de la cuenca del Pascua. Exs.: Entre Lago Leal y Quetru. R. Rodríguez y E. Teneb 4159 (CONC 166605).

Nothofagus dombeyi (Mirb.) Oerst "coigüe" Árbol nativo de Chile y Argentina. Se distribuye entre las regiones de O'Higgins y de Aisén. Dominante de los bosques siempreverdes de la cuenca del Baker. Exs.: Cochrane, Colonia Norte. Sector El Carmen. R.Rodríguez y E. Teneb 4099 (CONC 166606).

Nothofagus nitida (Phil.) Krasser

"coigüe de Chiloé"

Arbol endémico en Chile. Crece entre las regiones de Los Lagos y de Magallanes. Muy frecuente en las cercanías de Caleta Tortel, al sur de la cuenca del Baker. Exs.: Río Vagabundo. E. Teneb 453 (CONC 166711).

Nothofagus pumilio (Poepp. et Endl.) Krasser "lenga" Arbol nativo de Chile y Argentina. Se distribuye entre las regiones del Maule y de Magallanes. Es dominante en los bosques caducifolios de la cuenca del Baker y escaso en la cuenca del Pascua. Exs.: Lago Esmeralda. R. Rodríguez y E. Ruiz 4488 (CONC 166607).

\section{OnAGRACEAE}

Epilobium ciliatum Raf.

Hierba perenne, nativa de Chile y Argentina. Se distribuye entre las regiones de Atacama y de Magallanes. Frecuente en la cuenca del Baker. Exs.: Cochrane. Sector Los Mellizos. A. Jiménez 174 (CONC 166498).

Fuchsia magellanica Lam. "chilco" Arbusto con flores rojas vistosas, nativo de Chile y Argentina. Se distribuye entre las regiones de Valparaíso y de Magallanes. Frecuente en las cuencas del Baker y Pascua. Exs.: Camino de Cochrane a Tortel. Puente Nadi. R. Rodríguez y E. Teneb 4128 (CONC 166513).

\section{Oenothera magellanica Phil.}

Hierba anual, nativa de Chile y Argentina. En Chile se encuentra en la regiones de Valparaíso y Metropolitana, y en las regiones de Aisén y Magallanes. Poco frecuente en la cuenca del Baker. Exs.: Arenal en el río Baker, balsa Baker. E. Teneb 420 (CONC 166710).

Oenothera stricta Ledeb. ex Link "mitrún" Hierba anual, nativa de Chile y Argentina. Se distribuye entre las regiones de Atacama y de Magallanes. Es poco frecuente en sitios áridos de la cuenca del Baker. Exs.: Cochrane. Valle Grande. A. Marticorena 981 (CONC 166608).

\section{Plantaginaceae}

\section{Plantago australis Lam.}

Hierba perenne, nativa de Chile y Argentina. Se distribuye entre las regiones de Los Lagos y la de Magallanes. Frecuente en la cuenca del Baker. Exs.: Río Baker. Vagabundo. Puente Santo Domingo. E. Teneb 302 (CONC 166626).

\section{Plantago barbata G.Forst.}

Hierba perenne, nativa de Chile y Argentina. Se distribuye entre las regiones de Coquimbo y de Magallanes. Poco frecuente en arenales de la cuenca del Baker. Exs.: Cochrane. Nacimiento del Río Baker. C. Ramírez y O. Vidal s/n (CONC 166627). 
Plantago lanceolata L. "siete venas" Hierba perenne, introducida en Chile, originaria de Europa, casi cosmopolita. Se encuentra entre la Región de Antofagasta y la de Magallanes. Frecuente en sitios alterados de la cuenca del Baker. Exs.: Cochrane. Pasarela El Manzano. R. Rodríguez y E. Ruiz 4316 (CONC 166628).

\section{Plumbaginaceae}

Armeria maritima (Mill.) Willd. "manuelito" Hierba perenne, nativa de Chile y Argentina. Se distribuye entre las regiones de Coquimbo y la de Magallanes. Frecuente en sitios áridos de la cuenca del Baker. Exs.: Cochrane. El Salto. R. Rodríguez y E. Ruiz 4461 (CONC 166415).

\section{Polemoniaceae \\ Collomia biflora (Ruiz et Pav.) Brand}

"trinitri-lahuén"

Hierba anual, nativa en Chile y Argentina. Se distribuye entre las regiones de Valparaíso y de Magallanes. Poco frecuente en la cuenca del Baker. Exs.: Cochrane. El Salto. R. Rodríguez y E. Ruiz 4438 (CONC 166471).

Microsteris gracilis (Hook.) Greene Hierba anual, nativa de Chile y Argentina. Se distribuye entre las regiones de Atacama y la de Magallanes. Poco frecuente en la cuenca del Baker. Exs.: Cochrane. El Salto. R. Rodríguez y E. Ruiz 4433 (CONC 166586).

\section{Polygonaceae \\ Muehlenbeckia hastulata (Sm.) I.M.Johnst. "mollaca, quilo"} Arbusto trepador, nativo de Chile y Argentina. Se distribuye entre las regiones de Valparaíso y la de Aisén. Escaso en la cuenca del Baker. Exs.: Cochrane. Pasarela El Salto. C. Ramírez y O. Vidal s/n (CONC 166594).

\section{Polygonum aviculare L. "sanguinaria"} Hierba anual, introducida en Chile, originaria de Asia. Se distribuye entre las regiones de Atacama y la de Magallanes. Frecuente en la cuenca del Baker. Exs.: Cochrane. Lago Esmeralda. C. Ramírez y O. Vidal s/n (CONC 166635).

\section{Rumex acetosella $\mathrm{L}$.} "vinagrillo" Hierba perenne, introducida en Chile, originaria de Europa, casi cosmopolita. Se distribuye entre las regiones de Atacama a la de Magallanes. Muy frecuente en sitios alterados de la cuenca del Baker. Exs.: Cochrane. Sector El Manzano, curva Las Eses. R. Rodríguez y E. Ruiz 4306 (CONC 166658).

\section{Rumex pulcher $\mathrm{L}$.}

Hierba perenne, introducida en Chile, originaria de Europa. Se encuentra desde la Región de Valparaíso a la de Aisén. Poco frecuente en sitios alterados de la cuenca del Baker. Primera cita de especie para la Región de Aisén. Exs.: Cochrane. Pasarela El Salto. C. Ramírez y O. Vidal s/n (CONC 166659).

\section{Primulaceae}

Anagallis alternifolia Cav. "numáwe" Hierba anual, pequeña, nativa de Chile y Argentina. Se distribuye entre las regiones de Atacama y de Magallanes. Frecuente en la cuenca del río Baker. Exs.: Cochrane. Sector Lago Vargas. R. Rodríguez y E. Ruiz 4534 (CONC 166405).

\section{Proteaceae}

Embothrium coccineum J.R.Forst. et G.Forst. "Notro" Arbol con llamativas flores rojas, nativo de Chile y Argentina. Se distribuye entre las regiones del Maule y de Magallanes. Frecuente en forma de arbusto en ambas cuencas estudiadas. Exs.: Río Baker. El Saltón. R. Rodríguez y E. Teneb 4283 (CONC 166496).

Lomatia ferruginea (Cav.) R.Br. "fuinque" Árbol nativo de Chile y Argentina. Se distribuye entre las regiones del Maule y de Magallanes. Escaso en la cuenca del Baker y pero frecuente en la cuenca del Pascua. Exs.: Río Baker. El Saltón. R. Rodríguez y E. Teneb 4291 (CONC 166570).

\section{RANUNCULACEAE}

Anemone multifida Poir. "centella" Hierba perenne de vistosas flores blancas, nativa de Chile y Argentina. Se encuentra entre la Región del Bío-Bío y la de Magallanes. Abundante en pastizales y praderas de la cuenca del Baker. Exs.: Cochrane. Sector El Manzano, curva Las Eses. R. Rodríguez y E. Ruiz 4302 (CONC 166406).

Caltha appendiculata Pers. "maillico" Hierba perenne, nativa de Chile y Argentina. Se distribuye entre las regiones del Maule y de Magallanes. Abundante en turberas de la cuenca del Pascua. Exs.: Río Pascua. Angostura San Vicente. E. Teneb 364 (CONC 166446). 
Caltha dionaeifolia Hook.f.

Hierba perenne, nativa de Chile y Argentina. Se distribuye en las regiones de Aisén y de Magallanes. Frecuente en turberas de la cuenca del Pascua. Exs.: Río Pascua. Pampa Quiroz. E. Teneb y A. Jiménez 4234 (CONC 166447).

Caltha sagittata Cav. "maillico" Hierba perenne, nativa de Chile y Argentina. Se distribuye entre las regiones de Coquimbo y de Magallanes. Escasa en charcas y lagunas permanentes de la cuenca del Pascua. Exs.: Lago Quetru. C. Ramírez y O. Vidal s/n (CONC 166448).

\section{Ranunculus aquatilis $\mathrm{L}$.}

Hierba perenne, nativa de Chile y Argentina. Habita en casi todo Chile (Atacama a Magallanes). Es frecuente en charcas y lagunas permanentes de la cuenca del río Baker. Exs.: Cochrane. Valle Grande. A. Marticorena 974 (CONC 166646).

\section{Ranunculus minutiflorus Bertero ex Phil.}

"lumurka"

Hierba perenne, nativa de Chile y Argentina. Se distribuye entre las regiones de Coquimbo y de Magallanes. Frecuente en praderas y pastizales de la cuenca del Baker. Exs.: Cochrane. Sector Lago Vargas. R. Rodríguez y E. Ruiz 4535 (CONC 166647).

Ranunculus repens L. "botón de oro" Hierba perenne, introducida en Chile, originaria de Europa. Se encuentra desde la Región de Coquimbo a la de Magallanes. Es una maleza frecuente en praderas y pastizales de la cuenca del Baker. Exs.: Cochrane. Sector Puerto Bertrand. R. Rodríguez y E. Ruiz 4361 (CONC 166648).

\section{Ranunculus spegazzinii Lourtieg}

Hierba perenne, nativa de Chile y Argentina. Se distribuye en la regiones de Aisén y de Magallanes. Es escasa en la cuenca del Baker. Exs.: Chacabuco. Balseo Baker. C. Ramírez y O. Vidal s/n (CONC 166649).

\section{Ranunculus trullifolius Hook.f.}

Hierba perenne, nativa de Chile y Argentina. Se encuentra en la Región de Coquimbo, y entre las regiones de la Araucanía y la de Magallanes. Escasa en la cuenca del Baker. Exs.: Cochrane. Lago Vargas. A. Jiménez 26 (CONC 166650).

\section{RHamnaceae}

Colletia hystrix Clos "yaquil, yaqui" Arbusto nativo de Chile y Argentina. Se distribuye entre la Región de Atacama y Aisén. Dominante en determinadas formaciones en la cuenca del Baker. Exs.: Camino de Cochrane a Bertrand, Angostura El Manzano. R. Rodríguezy E. Teneb 4121 (CONC 166470).

Discaria chacaye (G.Don) Tortosa "chacay" Arbusto nativo de Chile y Argentina. Se distribuye entre la Región de Valparaíso y la de Magallanes. Abundante en determinadas formaciones vegetales de la cuenca del Baker. Exs.: Cochrane, Colonia Norte. Sector El Carmen. R. Rodríguez y E. Teneb 4100 (CONC 166485).

\section{ROSACEAE}

Acaena antarctica Hook.f.

Hierba perenne, nativa de Chile y Argentina. Se distribuye entre las regiones de Los Lagos y la de Magallanes. Es escasa en la cuenca del Baker. Exs.: Cochrane. Los Nadis. A. Jiménez 166(CONC 166388).

Acaena integerrima Gillies ex Hook. et Arn.

"cadillo"

Subarbusto nativo de Chile y Argentina. Se distribuye entre las regiones de Coquimbo y de Magallanes. Frecuente en sitios áridos de la cuenca del Baker. Exs.: Cochrane. Sector El Manzano. A. Marticorena 936 (CONC 166389).

Acaena magellanica (Lam.) Vahl

"pimpinela, abrojo"

Hierba perenne, nativa de Chile y Argentina. Se distribuye entre las regiones de Atacama y la de Magallanes. Frecuente en bosques deciduos y algunos pastizales de la cuenca del Baker. Exs.: Tortel. Desembocadura del río Baker. R. Rodríguez y E. Teneb 4200 (CONC 166390).

Acaena ovalifolia Ruiz et Pav. "cadillo" Hierba perenne, nativa de Chile y Argentina. Se distribuye entre las regiones de Coquimbo y de Magallanes. Frecuente en bosques deciduos y en algunos pastizales de ambas cuencas. Exs.: Cochrane, Colonia Norte. Sector El Carmen. R. Rodríguez y E. Teneb 4095 (CONC 166391).

Acaena pinnatifida Ruiz et Pav.

"pimpinela cimarrona"

Hierba perenne, nativa de Chile y Argentina. Se 
distribuye entre las regiones de Coquimbo y de Magallanes. Frecuente en pastizales de la cuenca del Baker. Exs.: Cochrane. Pasarela El Manzano. R. Rodríguez y E. Ruiz 4314 (CONC 166392).

\section{Acaena pumila Vahl}

"amor seco"

Hierba perenne, nativa de Chile y Argentina. Se distribuye entre las regiones de Los Lagos y de Magallanes. Escasa en turberas de ambas cuencas. Exs.: Tortel, parte alta, en una turbera. R. Rodríguez y E. Teneb 4077 (CONC 166393).

Aphanes neglecta (Rothm.) Rothm.

Hierba anual, nativa de Chile y Argentina. Se distribuye entre las regiones de O'Higgins y Aisén, Escasa en la cuenca del Baker. Este es el primer registro de la especie para la Región de Aisén. Exs.: Cochrane. Sector El Manzano. A. Marticorena 941 (CONC 166410).

Fragaria chiloensis (L.) Duch. "frutilla" Hierba perenne, nativa de Chile y Argentina. Se distribuye entre las regiones de O'Higgins y de Magallanes. Abundante en bosques decíduos de la cuenca del Baker, su fruto es comestible. Exs.: Cochrane. Puente río El Maitén. R. Rodríguez y E. Ruiz 4335 (CONC 166512).

Geum magellanicum Pers.

"llalante"

Hierba perenne, nativa de Chile y Argentina. Se distribuye entre las regiones de O'Higgins y Magallanes. Es frecuente en la cuenca del río Baker. Exs.: Cochrane. Pasarela El Manzano. R. Rodríguez y E. Ruiz 4309 (CONC 166529).

\section{Potentilla anserina L.}

"canelilla"

Hierba perenne, introducida en Chile, originaria de Europa. Se encuentra entre las regiones de Valparaíso y de Magallanes. Frecuente en humedales de la cuenca del Baker. Exs.: Cochrane. El Salto. A. Marticorena 958 (CONC 166642).

\section{Rosa rubiginosa $\mathrm{L}$.}

"mosqueta" Arbusto espinoso, introducido en Chile, originaria de Europa. Se encuentra en las regiones de Valparaíso, Metropolitana, Bío-Bío y Aysén. Abundante en variados ambientes de la cuenca del Baker, sus frutos son usados como alimento y en la industria farmacéutica. Exs.: Cochrane. El Salto, Valle Grande. R. Rodríguez y E. Teneb 4216 (CONC 166654).
Rubus geoides Sm.

"miñemiñe" Hierba perenne, nativa de Chile y Argentina. Se distribuye entre las regiones de la Araucanía y de Magallanes. Abundante en bosques y montes de ambas cuencas. Frutos comestibles. Exs.: Cochrane. Pasarela Nadis. E. Teneb 47 (CONC 166656).

Rubus radicans Cav. "miñemiñe" Hierba perenne, nativa de Chile y Argentina. En Chile se distribuye entre las regiones de la Araucanía y de Magallanes. Frecuente en bosques de ambas cuencas. Exs.: Entre Lago Leal y Quetru. R. Rodríguez y E. Teneb 4155 (CONC 166657).

Galium aparine L. RUBIACEAE "lengua de gato" Hierba anual, introducida en Chile, originaria de Europa, cosmopolita. Se encuentra entre las regiones de Atacama y de Magallanes. Frecuente en sitios sombríos de la cuenca del Baker. Exs.: Cochrane. El Salto. R. Rodríguez y E. Ruiz 4435 (CONC 166514).

\section{Galium fuegianum Hook.f.}

Hierba anual, nativa de Chile y Argentina. En Chile se distribuye entre las regiones de O'Higgins y de Magallanes. Escasa en la cuenca del Baker. Exs.: Lago Juncal. Sector Poco a Poco. R. Rodríguez y E. Teneb 4257 (CONC 166515).

Nertera granadensis (Mutis ex L.f.) Druce "coralito, rucachucao"

Hierba perenne, nativa de Chile y Argentina. Se encuentra entre las regiones de Coquimbo y de Magallanes. Escasa en ambas cuencas. Exs.: Cochrane. Sector Lago Vargas. R. Rodríguez y E. Ruiz 4532 (CONC 166603).

\section{Salicaceae}

Populus nigra L. var. italica Münchh. "álamo" Arbol introducido en Chile, originario de Europa es cultivado para la formación de avenidas y cortinas rompevientos. Es frecuente en lugares habitados de la cuenca del Baker. Exs. Cochrane, sector El Manzano (C. Ramírez*).

\section{Salix fragilis L.}

"sauce"

Arbol introducido en Chile, originario de Europa y sudoeste de Asia, es cultivado como cortavientos. Es abundante en las cercanías de las casas en la cuenca del Baker. Exs.: Cochrane. Sector Valle Castillo. R. Rodríguez y E. Teneb 4225 (CONC 166663). 
Salix viminalis $\mathrm{L}$.

"mimbre"

Arbusto introducido en Chile, originario de Europa y Asia, es cultivado como cortavientos. Es abundante en las cercanías de las casas en la cuenca del Baker. Exs.: Cochrane, Colonia Norte. Sector El Carmen. R.Rodríguez y E. Teneb 4098 (CONC 166664).

\section{Arjona pusilla Hook.f.}

\section{Santalaceae}

Hierba anual, nativa de Chile y Argentina. Se distribuye en las regiones de Aisén y Magallanes. Escasa en sitios áridos de la cuenca del Baker. Exs.: Cochrane, Balsa Colonia. R. Rodríguez y E. Ruiz 4472 (CONC 166414).

\section{Myoschilos oblonga Ruiz et Pav. \\ "codocoipo, orocoipo"}

Arbusto nativo de Chile y Argentina. Se distribuye entre las regiones de Coquimbo y de Magallanes. Abundante en bosques caducifolios de ambas cuencas. Exs.: Lago Juncal. Sector Poco a Poco. R. Rodríguez y E. Teneb 4255 (CONC 166597).

Nanodea muscosa Banks ex C.F.Gaertn.

Hierba perenne, nativa de Chile y Argentina. Se distribuye entre las regiones de Los Lagos y de Magallanes. Frecuente en turberas de ambas cuencas. Exs.: Río Pascua. Pampa Quiroz. E. Teneb y A. Jiménez 4235 (CONC 166601).

\section{Quinchamalium chilense Molina}

"quinchamalî"

Hierba perenne, nativa de Chile y Argentina. Se distribuye entre las regiones de Tarapacá y de Aisén. Frecuente en pastizales de ambas cuencas. Exs.: Cochrane. Sector El Manzano. A. Marticorena 946 (CONC 166645).

\section{SAXIFRAGACEAE}

\section{Saxifraga magellanica Poir.}

Hierba perenne, nativa de Chile y Argentina. Se distribuye entre las regiones del Maule y Aisén. Escasa en la cuenca del Baker. Exs.: Cochrane. Pasarela El Manzano. R. Rodríguez y E. Ruiz 4329 (CONC 166665).

\section{SCrophulariaceae}

\section{Bartsia trixago $\mathrm{L}$}

Hierba anual, introducida en Chile. Originaria de Europa. Se encuentra en la Región de Valparaíso y entre las regiones del Maule y la de la Araucanía.
Escasa en la cuenca del Baker. Este es el primer registro de la especie para la Región de Aisén. Exs.: Lago Vargas. E. Teneb 376 (CONC 166427).

\section{Euphrasia antarctica Benth.}

Hierba anual, nativa de Chile y Argentina. Se distribuye entre las regiones del Bío-Bío y de Magallanes. Frecuente en pastizales de la cuenca del Baker. Exs.: Cochrane. Valle Grande. A. Marticorena 968 (CONC 166505).

\section{Euphrasia cockayniana Petrie}

Hierba anual, nativa de Chile y Argentina. Se distribuye entre la Región de Aisén y la de Magallanes. Frecuente en pastizales de la cuenca del Baker. Exs.: Cochrane. Puerto Bertrand. C. Ramírez y O. Vidal s/n (CONC 166506).

\section{Euphrasia subexserta Benth.}

Hierba anual, endémica en Chile, donde se distribuye entre las regiones de Coquimbo y de Aisén. Es escasa en la cuenca del Baker. Primer registro de la especie para la Región de Aisén. Exs.: Cochrane. Pasarela El Salto. C. Ramírez y O. Vidal s/n (CONC 166507).

Mimulus glabratus Kunth "placa" Hierba anual, nativa en Chile y Argentina. Se distribuye entre las regiones de Atacama y de Magallanes. Escasa en la cuenca del Baker. Exs.: Cochrane. Sector Los Mellizos. A. Jiménez 148 (CONC 166587).

\section{Ourisia ruellioides (L.f.) Kuntze}

Hierba perenne con llamativas flores rojas, nativa en Chile y Argentina. Se distribuye entre las regiones de O'Higgins y de Magallanes. Escasa, se encuentra en riachuelos de la cuenca del Baker. Exs.: Cuesta Barrancoso, parte media. R. Rodríguez y E. Ruiz 4386 (CONC 166612).

Verbascum thapsus L. "hierba del paño" Hierba perenne, introducida en Chile, de origen euroasiático. Se encuentra entre las regiones de Valparaíso y Aisén. Abundante a orilla de caminos en la cuenca del Baker. Primera cita de esta especie para la Región de Aisén. Exs.: Lago Vargas. E. Teneb 377 (CONC 166708).

\section{Veronica peregrina $\mathrm{L}$.}

Hierba anual, introducida en Chile, originaria de Europa, cosmopolita. Se encuentra entre las regiones 
de Coquimbo y de Magallanes. Escasa en la cuenca del Baker. Exs.: Cochrane. Lago Vargas. E. Teneb 95 (CONC 166698).

\section{Veronica serpyllifolia $\mathrm{L}$.}

Hierba perenne, introducida en Chile, de origen euroasiático. En Chile se encuentra entre la Región de Valparaíso y la de Magallanes. Escasa en la cuenca del Baker. Exs.: Cochrane. Puerto Bertrand. A. Jiménez 10 (CONC 166699).

\section{STYLIDACEAE}

Phyllachne uliginosa J.R.Forst. et G.Forst. Hierba perenne, nativa de Chile y Argentina. Se distribuye en las regiones de Aisén y de Magallanes. Frecuente en turberas de la cuenca del Pascua. Exs.: Río Pascua. Pampa Quiroz. E. Teneb y A. Jiménez 4244 (CONC 166622).

Thymelaeaceae

Drapetes muscosus Banks ex Lam.

Hierba perenne, nativa de Chile y Argentina. Se distribuye entre las regiones de Los Lagos y de Magallanes. Escaso en turberas de la cuenca del Pascua. Exs.: RíoPascua. A. Jiménez 204(CONC 166489).

\section{Valeriana carnosa $\mathrm{Sm}$}

\section{VALERIANACEAE}

Hierba perenne, nativa de Chile y Argentina. Se distribuye entre la Región del Maule y la de Magallanes. Escasa en la cuenca del Baker. Exs.: Cochrane. Sector El Manzano, curva Las Eses. R. Rodríguez y E. Ruiz 4296 (CONC 166695).

\section{Valeriana clarioneifolia Phil.}

Hierba perenne, nativa de Chile y Argentina. En Chile se encuentra en la Región del Bío-Bío y la de Magallanes. Escasa en la cuenca del Baker. Primera cita de esta especie para la Región de Aisén. Exs.: Cochrane. Sector Los Mellizos. A. Jiménez 177 (CONC 166696).

Valeriana lapathifolia Vahl "huahuilque" Hierba perenne, nativa de Chile y Argentina. Se distribuye entre la Región de la Araucanía y la de Magallanes. Frecuente en ríos y riachuelos de ambas cuencas. Exs.: Cuesta Barrancoso, parte media. R. Rodríguez y E. Ruiz 4392 (CONC 166697).

ViolaceAe
Viola maculata Cav. "pilludén"
Hierba perenne, nativa de Chile y Argentina. Se

distribuye entre la regiones del Bío-Bío y la de Magallanes. Escasa en bosques caducifolios de la cuenca del Baker. Exs.: Cochrane. Puente río El Maitén. R. Rodríguez y E. Ruiz 4332 (CONC 166702).

\section{Viola reichei Skottsb.}

Hierba anual, nativa de Chile y Argentina. Se distribuye entre la regiones del Bío-Bío y la de Magallanes. Frecuente en bosques caducifolios de la cuenca del Baker. Exs.: Cochrane. Sector Puerto Bertrand. R. Rodríguez y E. Ruiz 4341 (CONC 166703).

Viola tridentata Menzies ex Ging.

Hierba perenne, nativa de Chile y Argentina. Se encuentra entre las regiones de Los Lagos y de Magallanes. Escasa en la cuenca del Pascua. Exs.: Río Pascua. A. Jiménez 227 (CONC 166704).

\section{WINTERACEAE}

Drimys winteri J.R.Forst. et G.Forst. "canelo" Arbol nativo de Chile y Argentina. Se distribuye entre la regiones de Coquimbo y la de Magallanes. Abundante en ambas cuencas estudiadas. Exs.: Río Pascua. Angostura San Vicente. R. Rodríguez y E. Teneb 4186 (CONC 166490).

\section{MAGNOLIOPHYTA - LILIOPSIDA}

\section{Asteliaceae}

Astelia pumila (G.Forst.) Gaudich.

Hierba perenne, nativa de Chile y Argentina. Se distribuye entre las regiones de Los Lagos y de Magallanes. Es dominante en turberas duriherbosas de ambas cuencas. Exs.: Tortel, parte alta, en una turbera. R. Rodríguez y E. Teneb 4083 (CONC 166417).

\section{Corsiaceae}

Arachnitis uniflora Phil. "flor de la araña" Hierba perenne, nativa de Chile y Argentina. Se distribuye entre la regiones del Maule y la de Magallanes. Escasa en la cuenca del Baker. Estado de conservación: Vulnerable, anteriormente ya había sido considerada como una especie en vías de extinción (Muñoz 1973). Exs.: Río Baker. Angostura Tamango. E. Teneb 287 (CONC 166411).

\section{Carex aphylla Kunth}

\section{Cyperaceae}

Hierba perenne, nativa de Chile y Argentina. Se distribuye entre las regiones de Coquimbo y Aisén. Escasa en la cuenca del Baker. Este es el primer 
registro de la especie para la Región de Aisén. Exs.: Cuesta Barrancoso, parte media. R. Rodríguez y E. Ruiz 4401 (CONC 166453).

\section{Carex barrosii Nelmes}

Hierba perenne, nativa de Chile y Argentina. Se distribuye entre las regiones del Bío-Bío y la de Magallanes. Escasa en la cuenca del Baker. Exs.: Río Baker. El Saltón. E. Teneb 121 (CONC 166454).

Carex curta Gooden.

Hierba perenne, nativa de Chile y Argentina. Se distribuye entre las regiones de Los Lagos y la de Magallanes. Frecuente en humedales de la cuenca del Baker. Exs.: Cochrane, entre Los Nadis y El Saltón. R. Rodríguez y E. Ruiz 4410 (CONC 166455).

\section{Carex darwinii Boott}

Hierba perenne, nativa de Chile y Argentina. Se distribuye entre las regiones de la Araucanía y la de Magallanes. Frecuente en humedales de ambas cuencas. Exs.: Cochrane. Colonia Sur. R. Rodríguez y E. Ruiz 4487 (CONC 166456).

Carex kingii (Boott) Reznicek

Hierba perenne, nativa de Chile y Argentina. Se distribuye en las regiones de Aisén y Magallanes. Escasa en la cuenca del Pascua. Este corresponde al primer registro de la especie para la Región de Aisén. Exs.: Sector Vagabundo. C. Ramírez y O. Vidal s/n (CONC 166457).

Carex macloviana D'Urv.

Hierba perenne, nativa de Chile y Argentina. Se distribuye entre las regiones del Maule y la de Magallanes. Frecuente en humedales de la cuenca del Baker. Exs.: Río Baker. El Saltón. E. Teneb 120 (CONC 166458).

\section{Carex magellanica Lam.}

Hierba perenne, nativa de Chile y Argentina. Se distribuye entre la Región de O’Higgins y la de Magallanes. Frecuente en turberas de ambas cuencas. Exs.: Cochrane. Pasarela río Vargas. R. Rodríguez y E. Ruiz 4518 (CONC 166459).

\section{Eleocharis melanostachys (D’Urv.) C.B.Clarke "quilmén"}

Hierba perenne, nativa de Chile y Argentina. Se distribuye entre las regiones de Atacama y de Magallanes. Frecuente en la cuenca del Baker. Exs.:
Cochrane. Puente Nadis. R. Rodríguez y E. Ruiz 4504 (CONC 166493).

Eleocharis pachycarpa E.Desv.

Hierba perenne, nativa en Chile y Argentina. En Chile se distribuye entre las regiones de Valparaíso y de Magallanes. Escasa en humedales de la cuenca del Baker. Exs.: Cochrane. Sector Lago Vargas. R. Rodríguez y E. Ruiz 4538 (CONC 166494).

Oreobolus obtusangulus Gaudich.

Hierba perenne, nativa de Chile y Argentina. Se distribuye entre las regiones del Bío-Bío y de Magallanes. Frecuente en turberas de ambas cuencas. Exs.: Cochrane. Sector Lago Vargas. R. Rodríguez y E. Ruiz 4529 (CONC 166609).

\section{Schoenoplectus californicus (C.A.Mey.) Soják "tromé, tahua-tahua"}

Hierba perenne, nativa en Chile y Argentina. Se distribuye entre las regiones de Atacama y la de Magallanes. Frecuente en lagos someros y lagunas de ambas cuencas. Exs.: Tortel. Desembocadura del río Baker. R. Rodríguez y E. Teneb 4203 (CONC 166669).

Schoenus andinus (Phil.) Pfeiff.

Hierba perenne, nativa de Chile y Argentina. Se distribuye entre las regiones de la Araucanía y la de Magallanes. Escasa en turberas de la cuenca del Baker pero abundante en turberas del río Pascua. Exs.: Río Pascua. Pascua 1. E. Teneb 129 (CONC 166668).

Scirpus inundatus (R.Br.) Poir. "cancán” Hierbaperenne, nativa de Chile y Argentina. Se distribuye entre lasregiones de Coquimbo y deMagallanes. Escasa en la cuenca del Baker. Exs.: Cochrane. Pasarela El Salto. A. Marticorena 963 (CONC 166670).

Uncinia phleoides (Cav.) Pers.

Hierba perenne, nativa de Chile y Argentina. Se distribuye entre las regiones de Coquimbo y Aisén. Escasa en bosques de la cuenca del Pascua. Exs.: Río Baker. Vagabundo. Puente Santo Domingo. E. Teneb 300 (CONC 166693).

Uncinia tenuis Poepp. ex Kunth

Hierba perenne, nativa de Chile y Argentina. Se distribuye entre las regiones de la Araucanía y la de Magallanes. Escasa en bosques de ambas cuencas. Exs.: Río Pascua. E. Teneb 150 (CONC 166694). 


\section{IRIDACEAE}

Herbertia lahue (Molina) Goldblatt "lahué" Hierba perenne, nativa de Chile y Argentina. Se distribuye entre las regiones de Valparaíso y Aisén. Escasa en la cuenca del Baker. Este corresponde al primer registro de la especie para la Región de Aisén. Estado de conservación: EN Peligro (Walter y Guillett 1998). Exs.: Cochrane. Lago Esmeralda. C. Ramírez y O. Vidal s/n (CONC 166538).

\section{Libertia chilensis (Molina) Gunckel} "tequel-tequel, calle-calle" Hierba perenne, nativa de Chile y Argentina. Se distribuye entre las regiones del Maule y la de Magallanes. Escasa en la cuenca del Baker. Exs.: Tortel. R. Rodríguez 814 (CONC 45561).

Solenomelus segethii (Phil.) Kuntze "anukale-kale" Hierba perenne, nativa de Chile y Argentina. Se distribuye entre las regiones de Coquimbo y de Magallanes. Escasa en la cuenca del Baker. Exs.: Cochrane. Valle Grande. A. Marticorena 970 (CONC 166684).

Tapeinia pumila (Hook.f.) Baillon

Hierba perenne, nativa de Chile y Argentina. Se distribuye entre las regiones de la Araucanía y de Magallanes. Frecuente en turberas de la cuenca del Pascua. Exs.: Río Pascua. Pampa Quiroz. E. Teneb y A. Jiménez 4242 (CONC 166686).

\section{JUNCACEAE \\ Juncus microcephalus Kunth}

Hierba perenne, nativa de Chile y Argentina. Se distribuye entre las regiones del Bío-Bío y de Magallanes. Frecuente en lagos someros y lagunas de ambas cuencas. Exs.: Tortel. Desembocadura del río Baker. R. Rodríguez y E. Teneb 4201 (CONC 166556).

\section{Juncus procerus E.Mey.}

"junquillo"

Hierba perenne, nativa de Chile y Argentina. Se distribuye entre las regiones del Bío-Bío y de Magallanes. Frecuente en lagos someros y lagunas de la cuenca del Baker. Exs.: Tortel. Desembocadura del río Baker. R. Rodríguez y E. Teneb 4202 (CONC 166557).

\section{Luzula racemosa Desv.}

Hierba perenne, nativa de Chile y Argentina. Se distribuye entre las regiones de Coquimbo y la de
Magallanes. Frecuente en sitios áridos de la cuenca del Baker. Exs.: Cochrane. Pasarela El Manzano. R. Rodríguez y E. Ruiz 4315 (CONC 166572).

\section{Marsippospermum grandiflorum (L.f.) Hook.} "junco canasto" Hierba perenne, nativa de Chile y Argentina. Se distribuye entre las regiones del Bío-Bío y de Magallanes. Frecuente en turberas de ambas cuencas. Exs.: Entre Lago Leal y Quetru. R. Rodríguez y E. Teneb 4162 (CONC 166581).

Rostkovia magellanica (Lam.) Hook.f. "junco" Hierba perenne, nativa de Chile y Argentina. Se distribuye entre las regiones de Los Lagos y de Magallanes. Frecuente en turberas de la cuenca del Pascua. Exs.: Fiordo Mitchel. A. Jiménez 113 (CONC 166655).

\section{JunCAGINACEAE}

Tetroncium magellanicum Willd.

Hierba perenne, nativa de Chile y Argentina. Se distribuye entre las regiones de la Araucanía y de Magallanes. Abundante junto a charcos de las turberas de ambas cuencas. Exs.: Cochrane. Sector Lago Vargas. R. Rodríguez y E. Ruiz 4531 (CONC 166689).

\section{LUZURIAGACEAE}

Luzuriaga marginata (Banks et Sol. ex Gaertn.) Benth. "quilineja"

Hierba perenne, nativa de Chile y Argentina. Se distribuye entre las regiones de Los Lagos y de Magallanes. Frecuente en bosques caducifolios y siempreverdes de ambas cuencas estudiadas. Exs.: Entre Lago Leal y Quetru. R. Rodríguez y E. Teneb 4154 (CONC 166573).

\section{ORCHIDACEAE}

Chloraea magellanica Hook.f.

Hierba perenne, nativa de Chile y Argentina. Se distribuye entre las regiones de la Araucanía y de Magallanes. Escasa en la cuenca del Baker. Exs.: Cochrane. Valle Grande. A. Marticorena 969 (CONC 166466).

Codonorchis lessonii (D’Urv.) Lindl. "palomita" Hierba perenne, nativa de Chile y Argentina. Se distribuye entre las regiones del Bío-Bío y de Magallanes. Es abundante en bosques decíduos de ambas cuencas. Exs.: Cuesta Barrancoso, parte alta. R. Rodríguez y E. Ruiz 4380 (CONC 166469). 
Gavilea araucana (Phil.) M.N.Correa

Hierba perenne, nativa de Chile y Argentina. Se distribuye entre las regiones del Bío-Bío y de Magallanes. Escasa en las cuencas del Baker y Pascua. Exs.: Sector Vagabundo. C. Ramírez y O. Vidal s/n (CONC 166523).

\section{Gavilea lutea (Pers.) M.N.Correa}

Hierba perenne, nativa en Chile y Argentina. Se distribuye entre las regiones de la Araucanía y de Magallanes. Frecuente en la cuenca del Baker. Exs.: Cochrane. Colonia Norte. E. Teneb 37 (CONC 166524).

\section{Gavilea odoratissima Poepp.}

Hierba perenne, nativa de Chile y Argentina. Se distribuye entre las regiones de la Araucanía y Aisén. Escasa en la cuenca del Baker. Exs.: Cuesta Barrancoso, parte alta. R. Rodríguez y E. Ruiz 4381 (CONC 166525).

\section{Gavilea supralabellata M.N.Correa}

Hierba perenne, nativa de Chile y Argentina. Se distribuye entre las regiones de Aisén y Magallanes. Escasa en la cuenca del Baker. Nuevo límite norte para la distribución de la especie. Exs.: Cochrane. Valle Grande. A. Marticorena 972 (CONC 166526).

\section{Philesiaceae}

Philesia magellanica J.F.Gmel. "coicopihue" Arbusto trepador, con vistosas flores rojas, nativo de Chile y Argentina. Se distribuye entre las regiones de Los Lagos y de Magallanes. Arbusto frecuente en los bosques siempreverdes en el sector sur de la cuenca del río Baker y abundante en la cuenca del río Pascua. Exs.: Río Pascua. Angostura San Vicente. R. Rodríguez y E. Teneb 4174 (CONC 166620).

\section{Poaceae}

Agrostis inconspicua Kunze ex E.Desv.

"chépica"

Hierba perenne, nativa de Chile y Argentina. Se distribuye entre la Región de Valparaíso y de Magallanes. Frecuente en praderas húmedas de la cuenca del Baker. Exs.: Río Baker. Colonia Sur. E. Teneb 338 (CONC 166398).

Agrostis leptotricha E.Desv.

Hierba perenne, nativa de Chile y Argentina. Se distribuye entre las regiones de Valparaíso y de Magallanes. Frecuente en praderas de la cuenca del Baker.Exs.: Río Baker. E. Caviedes B2.1 (CONC 166399).

\section{Agrostis stolonifera L.}

"chépica alemana"

Hierba perenne, introducida en Chile, originaria de Europa, cosmopolita. Se encuentra entre las regiones de Valparaíso y de Magallanes. Frecuente en la cuenca del Baker. Exs.: Cochrane, alrededores. A. Jiménez 159 (CONC 166400).

\section{Aira caryophyllea $\mathrm{L}$.}

Hierba anual, introducida en Chile, originaria de Europa. Se encuentra entre las regiones de Antofagasta y de Magallanes. Frecuente en suelos degradados de la cuenca del Baker. Exs.: Cochrane. Sector El Manzano, curva Las Eses. R. Rodríguez y E. Ruiz 4304 (CONC 166401).

\section{Aira praecox L.}

Hierba anual, introducida en Chile, originaria de Europa. Se encuentra en la Región de Magallanes, y en el Archipiélago de Juan Fernández. Frecuente en suelos degradados de la cuenca del Baker (C.Ramírez *).

\section{Alopecurus magellanicus Lam.}

Hierba perenne, nativa de Chile y Argentina. En Chile se distribuye entre la regiones del Bío-Bío y de Magallanes. Es frecuente en praderas húmedas de la cuenca del Baker. Exs.: Cochrane. Sector Puerto Bertrand. R. Rodríguez y E. Ruiz 4358 (CONC 166402).

\section{Alopecurus pratensis L.}

Hierba perenne, introducida en Chile, originaria de Europa. Se encuentra entre las regiones de Los Lagos y de Magallanes. Frecuente en praderas de la cuenca del Baker. Exs.: Cochrane. Colonia Sur. R. Rodríguez y E. Ruiz 4486 (CONC 166403).

Anthoxanthum juncifolium (Hackel) Veldk.

Hierba perenne, nativa de Chile y Argentina. Se distribuye entre las regiones de la Araucanía y de Aisén. Escasa en praderas de la cuenca del río Baker. Este es el primer registro de la especie para la Región de Aisén. Exs.: Río Baker. E. Caviedes B2.3 (CONC 166408).

\section{Anthoxanthum odoratum L}

"pasto oloroso" Hierba anual, introducida en Chile, originaria de Europa. Se encuentra entre las regiones del Maule y de Magallanes. Escasa en bosques de ambas cuencas. Exs.: Cuesta Barrancoso, parte alta. R. Rodríguez y E. Ruiz 4378 (CONC 166409). 
Arrhenatherum elatius (L.) P.Beauv. ex J.Presl et K.Presl subsp. bulbosus (Will.) Schübler et Martens "pasto cebolla"

Hierba perenne, introducida en Chile, originaria de Europa. Se encuentra entre las regiones de Valparaíso y Magallanes. Escasa en la cuenca del Baker. (C.Ramírez*)

Bromus catharticus Vahl "lanco, lancu" Hierba perenne, nativa de Chile y Argentina. Se distribuye entre las regiones de Atacama y la de Magallanes. Es frecuente en la cuenca del Baker. Exs.: Cochrane. Lago Esmeralda. E. Teneb 323 (CONC 166437).

Bromus cebadilla Steud.

"cebadilla" Hierba perenne, nativa de Chile y Argentina. Se distribuye entre las regiones de Atacama y de Magallanes. Es frecuente en la cuenca del Baker. Exs.: Cochrane. Sector Tres Lagos. E. Teneb 242 (CONC 166438).

\section{Bromus diandrus Roth}

Hierba anual, introducida en Chile, originaria del sur de Europa. Se encuentra desde la Región de Atacama hasta Aisén. Escasa en la cuenca del Baker. Este corresponde al primer registro de la especie para la Región de Aisén. Exs.: Cochrane. Pasarela El Manzano. R. Rodríguez y E. Ruiz 4323 (CONC 166439).

\section{Bromus hordeaceus L.}

"cebadilla"

Hierba anual, introducida en Chile, originaria de Europa. Se encuentra entre las regiones de Coquimbo y de Magallanes. Escasa en la cuenca del Baker. Exs.: Cochrane. Puerto Bertrand. A. Jiménez 61 (CONC 166440).

\section{Bromus secalinus L.}

Hierba anual, introducida en Chile, originaria de Europa. Se encuentra entre las regiones del Bío-Bío y de Aisén. Escasa en la cuenca del Baker. Exs.: Cochrane. Pasarela El Manzano. R. Rodríguez y E. Ruiz 4312 (CONC 166441).

\section{Calamagrostis suka Speg.}

Hierba perenne, nativa de Chile y Argentina. Se encuentra desde la Región de Los Lagos a la de Magallanes. Es frecuente en zonas xéricas, al norte de la cuenca del Baker. Exs.: Río Baker, límite arbóreo. E. Teneb 445 (CONC 166442).
Chusquea culeou E. Desv.

"colihue" Arbusto, nativo de Chile y Argentina. Se distribuye entre las regiones del Maule y Aisén. Abundante en bosques siempreverdes y caducifolios húmedos de la cuenca del Baker. Exs.: Camino de Cochrane a Tortel. Puente Nadis. R. Rodríguez y E. Teneb 4130 (CONC 166467).

Cortaderia pilosa (D’Urv.) Hackel "cortadera" Hierba perenne, nativa de Chile y Argentina. Se distribuye entre las regiones de O’Higgins y de Magallanes. Escasa en la cuenca del Baker. Exs.: Cochrane. Lago Vargas. E. Teneb 74 (CONC 166476).

Cortaderia selloana (Schult. et Schult.f.) Asch. et Graebn. "cortadera" Hierba perenne, nativa de Chile y Argentina. Se distribuye entre las regiones de Atacama y la de Magallanes. Escasa en la cuenca del Baker. Exs.: El Maitén. Sector río Neff. R. Rodríguez y E. Teneb 4278 (CONC 166477).

Cynosorus echinatus L. "cola de zorro" Hierba anual, introducida en Chile, originaria de Europa. Se encuentra entre las regiones de Valparaíso y de Magallanes. Escasa en la cuenca del Baker (C.Ramírez *).

Dactylis glomerata L. "pasto ovillo" Hierba perenne, forrajera, introducida en Chile, originaria de Europa. Se encuentra entre las regiones de Valparaíso y de Magallanes. Abundante en variados ambientes de la cuenca del Baker. Exs.: Cochrane. Colonia Sur. R. Rodríguez y E. Ruiz 4485 (CONC 166480).

Deschampsia elongata (Hook.) Munro ex Benth. Hierba perenne, nativa de Chile y Argentina. Se distribuye en las regiones de Aisén y Magallanes. Escasa en la cuenca del Baker. Exs.: Cuesta Barrancoso, parte media. R. Rodríguez y E. Ruiz 4403 (CONC 166481).

Deschampsia flexuosa (L.) Trin.

Hierba perenne, nativa de Chile y Argentina. Se distribuye entre las regiones del Bío-Bío y de Magallanes. Abundante en el sector norte de la cuenca del Baker. Exs.: Río Baker. El Saltón. E. Teneb 116 (CONC 166482). 


\section{Deschampsia laxa Phil.}

Hierba perenne, nativa de Chile y Argentina. Se distribuye entre las regiones de Los Lagos y de Magallanes. Frecuente en la cuenca del Baker. Exs.: Río Baker. E. Caviedes B2.5 (CONC 166483).

Elymus angulatus J.Presl. et C.Presl.

Hierba perenne, nativa de Chile y Argentina. Se encuentra desde la Región de Antofagasta a la Región de Magallanes. Es frecuente en diversos ambientes de la cuenca del Baker. Exs.: Río Baker. El Saltón. E. Teneb 119 (CONC 166495).

\section{Festuca gracillima Hook.f.}

"coirón"

Hierba perenne, nativa de Chile y Argentina. Se distribuye entre las regiones de Aisén y de Magallanes. Frecuente en pastizales de áreas áridas de la cuenca del Baker. Exs.: Confluencia de los ríos Baker y Ñadis. E. Teneb 240 (CONC 166508).

\section{Festuca magellanica Lam.}

Hierba perenne, nativa de Chile y Argentina. En Chile se distribuye entre las regiones de Coquimbo y de Magallanes. Escasa en la cuenca del Baker. Exs.: Cuesta Barrancoso, parte media. R. Rodríguez y E. Ruiz 4402 (CONC 166509).

Festuca pyrogea Speg.

"coirón"

Hierba perenne, nativa de Chile y Argentina. Se distribuye entre las regiones de la Araucanía y de Magallanes. Escasa en la cuenca del Baker. Exs.: Río Baker. Entre Nadis y Colonia. E. Teneb 211 (CONC 166510).

\section{Festuca rubra L.}

Hierba perenne, introducida en Chile, originaria de Europa. Se encuentra entre las regiones de Coquimbo y de Magallanes. Frecuente en la cuenca del Baker. Exs.: Cochrane. Pasarela río Nadis. R. Rodríguez y E. Ruiz 4415 (CONC 166511).

\section{Glyceria multiflora Steud}

Hierba perenne, nativa de Chile y Argentina. Se distribuye entre las regiones de O'Higgins y de Magallanes. Escasa en la cuenca del Baker. Exs.: Cochrane. El Salto. A. Marticorena 962 (CONC 166532).

Holcus lanatus L.

"pasto miel"

Hierba anual, introducida en Chile, originaria de Europa. Se encuentra entre las regiones de Coquimbo y de Magallanes. Hierba palatable, frecuente en la cuenca de Baker. Exs.: Cochrane. Puerto Bertrand. A. Jiménez 15 (CONC 166542).

Hordeum chilense Roem. et Schult.

Hierba perenne, nativa de Chile y Argentina. Se distribuye entre las regiones de Atacama al Bío-Bío y entre Aisén y Magallanes. Es frecuente en praderas húmedas de la cuenca del Baker. Exs.: Arenal en el río Baker, balsa Baker. E. Teneb 426 (CONC 166709).

Hordeum murinum L. "flechilla" Hierba perenne, introducida en Chile, originaria de Europa. Se encuentra entre las regiones de Antofagasta y Aisén. Es frecuente en praderas húmedas de la cuenca del Baker. Este es el primer registro de la especie para la Región de Aisén. Exs.: Cochrane, en una plantación. A. Jiménez 157 (CONC 166543).

Jarava nana (Speg.) Peñail.

Hierba perenne, nativa de Chile y Argentina. Es frecuente en sitios áridos, al norte de la cuenca del Baker. Se encuentra sólo en Aisén y éste constituye primer registro de la especie para Chile. Exs.: Río Baker. Angostura Tamango. E. Teneb 258 (CONC 166554).

Jarava psylantha (Speg.) Peñail.

Hierba perenne, nativa de Chile y Argentina. Se distribuye en las regiones de Aisén y Magallanes. Escasa en la cuenca del río Baker. Primera cita de la especie para la Región de Aisén. Exs.: Cochrane. Sector Los Mellizos. A. Jiménez 176b (CONC 166555).

Lolium perenne L. "ballica inglesa" Hierba perenne, introducida en Chile, originaria de Europa. En Chile se encuentra entre la Región de Coquimbo y la de Magallanes. Abundante en praderas de la cuenca del Baker. Exs.: Cochrane. Baker Norte. C. Ramírez y O. Vidal s/n (CONC 166569).

Nassella gigantea (Steud.) M.Muñoz “coirón” Hierba perenne, nativa de Chile y Argentina. Se distribuye entre las regiones de Coquimbo y de Aisén. Escasa en la cuenca del Baker. Exs.: Cochrane, Balsa Colonia. R. Rodríguez y E. Ruiz 4462 (CONC 166602).

Ortachne rariflora (Hook.f.) Hughes

Hierba perenne, nativa de Chile y Argentina. Se distribuye entre la Región de Los Lagos y la de Magallanes. Escasa en la cuenca del Pascua. Exs.: 
Río Pascua. Angostura San Vicente. E. Teneb 359 (CONC 166610).

\section{Phleum alpinum $\mathrm{L}$.}

Hierba perenne, nativa de Chile y Argentina. Se distribuye entre la Región de Coquimbo y la de Magallanes. Es frecuente en praderas de la cuenca del Baker. Exs.: Cochrane. Colonia Norte. E. Teneb 20 (CONC 166621).

\section{Poa annua L.}

"pasto piojillo" Hierba anual, introducida en Chile, originaria de Europa. Se encuentra entre la Región de Antofagasta y la de Magallanes. Es abundante en praderas húmedas de la cuenca del Baker (C.Ramírez *).

\section{Poa denudata Steud.}

Hierba anual, nativa en Chile y Argentina. Se distribuye entre la Región de Los Lagos y Aisén. Escasa en la cuenca del Baker. Primera referencia de la especie para la Región de Aisén. Exs.: Cochrane. Predio Pucchi. R, Rodríguez y E. Ruiz 4429 (CONC 166629).

\section{Poa glauca Vahl}

Hierba anual, introducida en Chile, originaria de Europa. Se encuentra entre la Región de Aisén y la de Magallanes. Escasa en la cuenca del Baker. Primera cita de la especie para la Región de Aisén. Exs.: Cochrane. Lago Chacabuco. A. Jiménez 52 (CONC 166630).

\section{Poa nemoralis L.}

Hierba anual, introducida en Chile, originaria de Europa. Se encuentra entre la Región de Aisén y la de Magallanes. Abundante en áreas bajo presión ganadera de la cuenca del Baker. Primera cita de la especie para la Region de Aisén. Exs.: Río Baker. Angostura Tamango. E. Teneb 264 (CONC 166631).

\section{Poa pratensis L.}

Hierba perenne, introducida en Chile, originaria de Europa. Se encuentra entre la Región de Atacama y la de Magallanes. Es abundante en praderas húmedas de la cuenca del Baker (C.Ramírez*).

\section{Poa resinulosa Nees ex Steud}

Hierba anual, nativa en Chile y Argentina. Se distribuye entre las regiones de Aisén y Magallanes. Escasa en la cuenca del Baker. Exs.: Cochrane. Predio Senén Rivera. R. Rodríguez y E. Ruiz 4370 (CONC 166632).
Poa spiciformis (Steud.) Hauman et Parodi Hierba anual, nativa de Chile y Argentina. Se distribuye desde la Región de Los Lagos hasta la de Magallanes. Escasa en la cuenca del Baker. Exs.: Cochrane. Colonia Sur. R. Rodríguez y E. Ruiz 4484 (CONC 166633).

Polypogon elongatus Kunth.

Hierba perenne, nativa de Chile y Argentina. Se distribuye entre las regiones Metropolitana y de los Lagos. Escaso en ambas cuencas. Primer registro de la especie para la región de Aisén. Exs.: Cochrane. Pasarela Lago Vargas. A. Jiménez 73 (CONC 166636).

Polypogon monspeliensis (L.) Desf.

Hierba anual, introducida en Chile, originaria de Europa. Se encuentra desde la Región de Tarapacá hasta la de Aisén. Escasa en ambas cuencas. Primera cita de la especie para la Región de Aisén. Exs.: Cochrane. Lago Vargas. A. Jiménez 22 (CONC 166637).

Rytidosperma virescens (E.Desv.) Nicora Hierba perenne, nativa de Chile y Argentina. Se distribuye desde la Región de Coquimbo a la de Magallanes. Frecuente en sitios áridos, al norte de la cuenca del Baker. Exs.: Río Baker. Angostura Tamango. E. Teneb 255 (CONC 166661).

Trisetum spicatum (L.) K.Richt.

Hierba perenne, introducido es Chile. Nativo de europa y con amplia distribución en el mundo. Se encuentra entre la Región de Valparaíso y la de Magallanes. Frecuente en la cuenca del Baker. Exs.: Cochrane. Pasarela El Manzano. R. Rodríguez y E. Ruiz 4317 (CONC 166692).

Vulpia antucensis Trin.

Hierba anual, nativa de Chile y Argentina. Se distribuye entre las regiones del Bío-Bío a la de Magallanes. Frecuente en la cuenca del Baker. Exs.: Cochrane. El Salto. A. Marticorena 959 (CONC 166705).

\section{Vulpia bromoides (L.) Gray}

Hierba anual, introducida en Chile, originaria de Europa. Se encuentra desde la Región de Coquimbo hasta la de Magallanes. Frecuente en la cuenca del Baker. Exs.: Río Baker. Angostura Tamango. E. Teneb 256 (CONC 166706). 


\section{Potamogetonaceae}

Potamogeton linguatus Hagstr.

"huiro, ahuiranque"

Hierba perenne, acuática, nativa de Chile y Argentina. Se distribuye entre las regiones del BíoBío y la de Magallanes. Frecuente en lagunas someras de ambas cuencas. Exs.: Cochrane. Colonia Sur. R. Rodríguez y E. Ruiz 4474 (CONC 166641).

\section{AGRADECIMIENTOS}

A Carlos Baeza, Clodomiro Marticorena, María Negritto y Eduardo Ruiz, del Departamento de Botánica de la Universidad de Concepción por el apoyo en las determinaciones taxonómicas y datos de distribución. A Alejandra Jiménez y Osvaldo Vidal por el apoyo en terreno. Al proyecto Determinación de Línea Base del Proyecto Hidroeléctrico Aisén. Al Laboratorio de Geomática del Instituto Manejo Forestal y al Laboratorio de Geobotánica, ambos de la Universidad Austral de Chile y a la base de datos CONC del proyecto Flora de Chile.

\section{BIBLIOGRAFIA}

Baeza, M., E. Barrera, J. Flores, C. Ramírez \& R. Rodríguez. 1998. Categorías de conservación de Pteridophyta nativas de Chile. Boletín del Museo Nacional de Historia Natural 47: 23-46.

CRonquist, A. 1981. An integrated system of classification of flowering plants. New York. Columbia University Press. xviii, 1262 pp.

Dollenz, O. 2003. Maytenus boaria Molina (Celastraceae) en la Región de Magallanes, Chile. Anales del Instituto de la Patagonia 31: 87-89.

DusÉn, P. 1903. The vegetation of western Patagonia. Report of the Princeton University Expedition to Patagonia 8, Bot.: 1-33

Dusén, P. \& F. Neger. 1908. Chilenisch-patagonische Charakterpflanzen. Vegetationsbilder 6(8): taf. 43-48.

Espinosa, M.R. 1935. Plantas de Aysén. Boletín del Museo Nacional de Historia Natural 14: 65-82.

GAJARDO, R. 1994. Vegetación natural de Chile. Clasificación y distribución geográfica. Editorial Universitaria. Santiago, Chile. 165 pp.

GunCKEL, H. 1970. Algunas especies vegetales de la Patagonia occidental chilena coleccionadas por la Segunda Expedición Japonesa. Boletín Universidad de Chile 106: $17-28$.

Hambleton, S. 1936. La vegetación del canal y río Baker (Patagonia occidental). Revista Argentia de Agronomía 3(3): 159-173.

Hechenleitner, P., M. Gardner,P. Thomas, C. Echeverría, B. Escobar, P. Brownless \& C. Martínez. 2005.
Plantas amenazadas del Centro-Sur de Chile. Universidad Austral de Chile. Real Jardín Botánico de Edimburgo. $187 \mathrm{pp}$.

Looser, G. 1945. Rápida excursión botánica a la Patagonia chilena. Revista de Geografía Americana 24(145): 191-200.

Luebert, F. \& P. Puiscoff. 2006. Sinopsis bioclimática y vegetacional de Chile. Editorial Universitaria. Santigo de Chile. 316 pp.

M ACloskie, G. 1903-06. Pteridophyta, ferns and fernlike plants. Flora Patagonica. Flowering plants. Analysis of orders and families. Collectors and bibliography. Topography. Character and origen of the Patagonian flora. Report of the Princeton University Expedition to Patagonia 8, Botany: 127-920.

M acloskie, G \& P. Dusén. 1915. Revision of the Flora Patagonia. Report of the Princeton University Expedition to Patagonia 8, Botany, Suppl.: 1-307.

Marticorena, C. \& M. Quezada. 1985. Catálogo de la flora vascular de Chile. Gayana Botánica 42 (1-2): 5-155.

M ATthei, O. 1995. Manual de las malezas que crecen en Chile. Alfabeta Impresores. Santiago, Chile. 543 pp.

MuÑoz, C. 1973. Chile: plantas en extinción. Santiago, 248 pp., 41 figs. y 31 lám.

Neger, F.W. 1901. Pflanzengeographisches aus den südlichen Anden und Patagonien. Botanische Jahrbücher Systematik 28: 231-258.

PARODI, L.R. 1959. Enciclopedia argentina de agricultura y jardinería. I. Descripción de las plantas cultivadas. Buenos Aires. 931 pp.

Pisano, E. 1972. Algunos resultados botánicos de la II expedición neozelandeza al hielo nor-patagónico, 1971-72. Anales del Instituto de la Patagonia 3(12): $131-160$.

Pisano, E. 1981. Bosquejo fitogeográfico de Fuego-Patagonia. Anales del Instituto de la Patagonia 12: 159-171.

PisAno,E. 1988. Sectorización fitogeográfica del archipiélago sud patagónico-fueguino: II. Vegetación y flora vascular del área del Parque Nacional "Laguna San Rafael", Aysén (Chile). Anales. Inst. Patagonia Ser. Ci. Nat. Vol. 18: 5-34.

ReICHe, K. 1907. Grundzüge der pflanzenverbreitung in Chile.Die Vegetation der Erde. 8: 1-394.

Rodríguez, R., E. Ruiz \& J.P.Elissetche. 2005. Arboles en Chile. Editorial Universidad de Concepción. Santiago, Chile. 183 pp.

SEKI, T. 1968. Sobre las plantas del nor-oeste de Patagonia. Proceedings Japan Society of Plant Taxonomy 2(3): 30-36.

Sкотtsberg, C. 1910. Botanische Ergebnisse der schwedischen Expedition nach Patagonien und dem Feuerlande 1907-1909. I. Übersicht über die wichtigsten Pflanzenformationen Südamerikas S. von $41^{\circ}$, ihre geographische Verbreitung und Beziehungen zum Klima. Kongl. Svenska Vetenskapsakademiens Handlingar 46(3): 1-28. 
Skottsberg, C. 1916. Botanische Ergebnisse der schwedischen Expedition nach Patagonien und dem Feuerlande 1907-1909. V. Die Vegetationsverhältnisse längs der Cordillera de los Andes S. von $41^{\circ} \mathrm{S}$. Br. Ein Beitrag zur Kenntnis der Vegetation in Chiloé, Westpatagonien, dem andinen Patagonien und Feuerland. Kongl. Svenska Vetenskapsakademiens Handlingar 56(5): 1-366.

Skottsberg, C. 1921. Algunos resultados botánicos obtenidos durante la campaña de la comisión sueca en los territorios australes de Chile y Argentina, en los años 1908-1909. Revista Chilena de Historia Natural 25: 474-494.

Skottsberg, C. 1923. Zur Geffässpflanzenflora Westpatagoniens. Götheborgs Kungl. Vetenskaps och Vitterheta Samhällets Handlingar, ser. 4, 28(3): 1-29.

StefFen, H. 1904. Bericht über eine Reise in das chilenische Fjordgebiet nordlich vom $48^{\circ} \mathrm{S}$. Verhandlungen des Deutschen Wissenschaftlichen Vereins zu Santiago de Chile 5 (1): 37-116.

Teillier, S. \& C. Marticorena. 2002. Riqueza florística del Parque Nacional Laguna San Rafael, XI Región, Chile. Boletín del Museo Nacional de Historia Natural de Santiago 51: 43-73.

Torres-Mura, J.C. \& G. Rojas (Eds.). 2004. Historia Natural, Reserva Nacional, Lago Jeinimeni. Proyecto Biodiversidad de Aysén-CONAF XI Región. 88 pp.

TurRIL, W.B. 1919. Botanical results of Swedish South American and Antarstic Expeditions. Bulletin of Miscellaneous Informations. 1919: 268-279.

WALter, K.S. \& Gillett, H.J. (Eds.) (1998). 1997 IUCN Red List of Threatened Plants. Compiled by the World Conservation Monitoring Centre. IUCNThe World Conservation Union, Gland, Switzerland an Cambridge, UK. lxiv + 862 pp. 